\title{
Transient biouptake flux and accumulation by micro-organisms. The case of two types of sites with Langmuir adsorption
} Josep Galceran $^{\mathbf{a}}$, Josep Monné ${ }^{\mathbf{a}}$, Jaume Puy ${ }^{\mathbf{a}}$ and Herman P. van Leeuwen ${ }^{\mathbf{b}}$

${ }^{\mathrm{a}}$ Departament de Química, Universitat de Lleida, Rovira Roure 191, 25198, Lleida, Spain

${ }^{\mathrm{b}}$ Laboratory of Physical Chemistry and Colloid Science, Wageningen University, Dreijenplein 6, 6703 HB Wageningen, The Netherlands

* Corresponding author. e-mail address: jpuy@quimica.udl.es

\section{Abstract:}

The uptake of a chemical species by an aquatic micro-organism is modelled considering two kinds of sites where Langmuirian adsorption is followed by first order internalisation kinetics. Simpler models, such as only one internalisation route (while most of the adsorption takes places on non-internalising sites) or a linear isotherm for adsorption on one or both sites, become limiting cases of this double-Langmuirian model. The model considers the sites located on the spherical (or semi-spherical) surface of the organism, and takes diffusion from the medium into explicit account. The numerical solution for the internalisation flux shows a maximum. We provide an estimate for the time needed to reach a certain proximity to steady state. The transient solution confirms that the analytical expressions for the steady-state flux are usually valid and that the accumulated amounts reflect the impact of the short-time uptake. The Instantaneous Steady-State Approximation (ISSA), where an intercept of the linear regression of accumulated amount as a function of time is interpreted as an adsorbed amount, can be critically assessed with the transient numerical code for two cases: i) when the total burden of metal on the cell is the input data and ii) when an extraction procedure provides further information on the adsorbed and internalised amount. 
Keywords: uptake, bioaccumulation, Chlorella kesslerii, unsteady regime, mathematical model

\section{Introduction}

A thorough understanding of the uptake of species (be it a nutrient or a toxicant) by organisms (e.g. phytoplankton) is essential for a number of purposes, ranging from environmental regulation to basic bioenvironmental science (Galceran and van Leeuwen 2004; Wilkinson and Buffle 2004). A key issue is the realisation that tackling data interpretation with appropriate rigour requires that experiments are conducted in perfectly well-defined media (Slaveykova and Wilkinson 2002;Sunda and Huntsman 1992). In this respect, models can help to identify the most relevant phenomena and assess suitable methods for retrieving the characteristic parameters of the system.

Some well-known models, such as the Free Ion Activity Model (FIAM) or the Biotic Ligand Model (BLM) discard the influence of diffusion on the uptake flux (i.e. on the amount of the relevant species arriving at the surface of the organism per unit of time and area) and can be classified as “equilibrium” approaches (Campbell 1995;Campbell et al. 2002;Hudson 1998; Tessier et al. 1994). However, this simplification is not valid in all situations as shown in several recent reports (Fortin and Campbell 2000;Hassler and Wilkinson 2003;Hudson and Morel 1990; Jansen et al. 2002). Taking diffusion fully into account, we have recently analysed the particular case where uptake is described by linear adsorption to the sites on the surface of the organism followed by first order internalisation (Galceran et al. 2004). 
Apart from diffusion, another issue to consider is the number of types of sites and their nature (in terms of both, their physiological activity and the specific adsorption isotherm). In order to interpret the uptake of Zn on Emiliana huxleyi, Sunda and Huntsman (Sunda and Huntsman 1992) introduced the distinction of two types of adsorbing-internalising sites where adsorption was described by a Langmuir isotherm on one of them while the adsorption on the other was described by a linear isotherm. (A Langmuirian isotherm followed by first order internalisation is equivalent to a Michaelis-Menten description (Galceran and van Leeuwen 2004) of a steady-state regime for the transporter sites in the membrane). In their interpretation of $\mathrm{Pb}$ uptake by Chlorella kesslerii, Slaveykova and Wilkinson (Slaveykova and Wilkinson 2002) also used two kinds of Langmuirian sites, one of which was physiologically active (i.e. internalisation follows adsorption) and the other not (i.e. adsorption-only). Our previous linear model (Galceran et al. 2004)(Galceran and van Leeuwen 2004) could embrace any number of physiologically active and non-active sites (by means of averaged parameters) as long as the coverages were small enough for linear isotherms to apply for each kind of sites. However, a linear adsorption isotherm cannot account for saturation effects such as those observed in the experiments of Slaveykova and Wilkinson (Slaveykova and Wilkinson 2002) and expected to occur due to the finite number of transporters. Here we improve on our previous linear model by use of the Langmuir isotherm with two kinds of adsorption sites (extension to a larger number of sites is straightforward). Such a model can identify parameters that correspond to limiting situations while describing the range of applicability of the simplistic FIAM, BLM and linear model. 
A third issue is the consideration of the geometry and size of the micro-organism. Here we consider the simplest, spherical, shape; the model can be straightforwardly adapted to include sites lying on a semi-spherical active surface. As a consequence of the small radius of aquatic micro-organisms, steady state is attained rapidly; consequently most available experimental techniques are not able to follow the transient regime at the beginning of the experiment nor to probe fluctuations of solute concentration ("patchy" regions) (Lippemeier et al. 2001).

Our model is used to evaluate the validity of the usual steady-state approach (i.e. disregarding the transient evolution). Not only is there the question of the flux value, but also what is the impact of the transient regime on the accumulated amount, the importance of which has been recently pointed out (Galceran et al. 2004). This knowledge allows a critical assessment of the limitations in recently suggested procedures (based on the Instantaneous Steady-State Approximation, ISSA) to retrieve system parameters from the intercept of accumulated amounts versus time plots (Galceran et al. 2004;Hassler et al. 2004b;Hassler et al. 2004a;Hassler and Wilkinson 2003;Slaveykova and Wilkinson 2002; Wilkinson et al. 2002).

\section{1.- Mathematical formulation of the model.}

Let $\mathrm{M}$ be a chemical species (present in the bulk of the medium with a concentration $c_{\mathrm{M}}^{*}$ ) which diffuses towards the spherical surface of the micro-organism whose radius is $r_{0}$ (see Fig 1). Assuming diffusion is the only relevant transport phenomenon, the continuity equation reads

$$
\frac{\partial c_{\mathrm{M}}(r, t)}{\partial t}=D_{\mathrm{M}}\left(\frac{\partial^{2} c_{\mathrm{M}}(r, t)}{\partial r^{2}}+\frac{2}{r} \frac{\partial c_{\mathrm{M}}(r, t)}{\partial r}\right)
$$


In the most general case considered here, $\mathrm{M}$ is adsorbed onto two kinds of sites, labelled 1 and 2. Each adsorption process is assumed to be fast enough (when compared with diffusion or the internalisation processes) to be described by a Langmuir equilibrium isotherm that relates the coverage of each kind of sites $\left(\Gamma_{1}(t) / \Gamma_{\max , 1}\right.$ and $\left.\Gamma_{2}(t) / \Gamma_{\max , 2}\right)$ and the concentration of $\mathrm{M}$ at the organism surface $c_{\mathrm{M}}\left(r_{0}, t\right)$ :

$\frac{\Gamma_{1}(t)}{\Gamma_{\max , 1}}=\frac{c_{\mathrm{M}}\left(r_{0}, t\right)}{K_{\mathrm{M}, 1}+c_{\mathrm{M}}\left(r_{0}, t\right)}$

$\frac{\Gamma_{2}(t)}{\Gamma_{\max , 2}}=\frac{c_{\mathrm{M}}\left(r_{0}, t\right)}{K_{\mathrm{M}, 2}+c_{\mathrm{M}}\left(r_{0}, t\right)}$

where $\Gamma_{1}$ and $\Gamma_{2}$ stand for the surface concentrations and $K_{\mathrm{M}, 1}$ and $K_{\mathrm{M}, 2}$ are adsorption constants in the Michaelis-Menten-like expression of the Langmuir isotherm (Galceran and van Leeuwen 2004;van Leeuwen and Pinheiro 2001).

We assume that $\mathrm{M}$ is taken up following a first order kinetic process (Galceran and van Leeuwen 2004;Pinheiro et al. 2004;van Leeuwen and Pinheiro 2001; Whitfield and Turner 1979) at each of the sites (where $M$ has been adsorbed) with internalisation rate constants $k_{1}$ and $k_{2}$, giving rise to the uptake flux:

$$
J_{\mathrm{u}}(t)=J_{\mathrm{u}, 1}(t)+J_{\mathrm{u}, 2}(t)=k_{1} \Gamma_{1}(t)+k_{2} \Gamma_{2}(t)
$$

As is well known, for $c_{\mathrm{M}}\left(r_{0}, t\right)$ much less than $K_{\mathrm{M}, 1}$ and $K_{\mathrm{M}, 2}$, the Langmuirian isotherms (2) and (3) tend towards the linear ones. On the other hand, when $c_{\mathrm{M}}\left(r_{0}, t\right)$ is much larger than $K_{\mathrm{M}, 1}$ and $K_{\mathrm{M}, 2}$, the saturation of active sites (membrane transporters) leads to a maximum for the uptake flux $J_{\mathrm{u}}$, which is not considered by the linear isotherm case. 
As expected from the presence of non-linear terms (Langmuirian isotherms) in the boundary conditions, no analytical solution for the stated problem (eqn. (1) with boundary conditions (A-1)-(A-3)) is available to our knowledge, so a numerical strategy is applied here (see details in Appendix A). Once the integral eqn. (A-6) is solved, any magnitude of the system can be computed. In particular, the incoming diffusion (or mass transport) flux

$$
J_{\mathrm{m}}(t) \equiv D_{\mathrm{M}}\left(\frac{\partial c_{\mathrm{M}}(r, t)}{\partial r}\right)_{r=r_{0}}
$$

has been selected in this work as a relevant response function. Notice that a positive sign is assigned to fluxes towards the micro-organism.

The cumulative supplied amount (via mass transfer, labelled here $\Phi_{\mathrm{m}}$ ) at any time, can be computed as the internalised amount, $\Phi_{\mathrm{u}}$, plus the adsorbed amount. Thus, assuming $\Gamma_{1}(0)=\Gamma_{2}(0)=0$, we have:

$$
\Phi_{\mathrm{m}}(t)=\int_{0}^{t} J_{\mathrm{m}}(\tau) d \tau=\Phi_{\mathrm{u}}(t)+\Gamma(t)=\int_{0}^{t} J_{\mathrm{u}}(\tau) d \tau+\Gamma_{1}(t)+\Gamma_{2}(t)
$$

\section{2.- The eventual steady-state regime}

Let us now consider the simple case of steady state, which is practically reached within a very short time for small enough $r_{0}$. In this case, the balance of fluxes $J_{\mathrm{m}}=J_{\mathrm{u}}$ can be written as (van Leeuwen and Pinheiro 2001):

$$
D_{\mathrm{M}} \frac{c_{\mathrm{M}}^{*}-c_{\mathrm{M}}\left(r_{0}\right)}{r_{0}}=k_{1} \Gamma_{\max , 1} \frac{c_{\mathrm{M}}\left(r_{0}\right)}{K_{\mathrm{M}, 1}+c_{\mathrm{M}}\left(r_{0}\right)}+k_{2} \Gamma_{\max , 2} \frac{c_{\mathrm{M}}\left(r_{0}\right)}{K_{\mathrm{M}, 2}+c_{\mathrm{M}}\left(r_{0}\right)}
$$

The impact of the parameters on the solution of this cubic equation for the unknown $c_{\mathrm{M}}\left(r_{0}\right)$ has been examined elsewhere by means of a graphical procedure (Galceran and van Leeuwen 2004). The key point in the procedure is to locate the intersection between 
the straight line of the left hand side in (7) (labelled $J_{\mathrm{m}}^{\mathrm{dSS}}$, the diffusive steady- state flux corresponding to the diffusion profile having reached the steady state with any given concentration value at the surface) and $J_{\mathrm{u}}$, when plotting uptake fluxes against varying concentrations $c_{\mathrm{M}}\left(r_{0}\right)$ at the surface.

The three solutions for $c_{\mathrm{M}}\left(r_{0}\right)$ can be found graphically (Galceran and van Leeuwen 2004) at each intersection of $J_{\mathrm{m}}^{\mathrm{dSS}}$ with the curve for $J_{\mathrm{u}}=J_{\mathrm{u}, 1}+J_{\mathrm{u}, 2}$ (which is the addition of two hyperbolae with their corresponding vertical asymptotes at $c_{\mathrm{M}}\left(r_{0}\right)=-K_{\mathrm{M}, 1}$ and at $\left.c_{\mathrm{M}}\left(r_{0}\right)=-K_{\mathrm{M}, 2}\right)$. Due to the positive character of all the physical constants, two of the solutions of (7) are negative, while the positive solution (which will be denoted $c_{\mathrm{M}}^{\mathrm{SS}}$ ) is the physically meaningful one. Fig 2 plots the positive range of $c_{\mathrm{M}}\left(r_{0}\right)$ and shows how the graphical procedure leads to the determination of three $c_{\mathrm{M}}^{\mathrm{SS}}$ values for three different

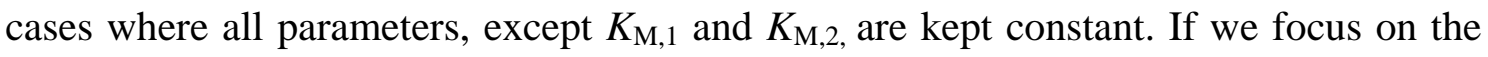
case with $K_{\mathrm{M}, 1} \neq K_{\mathrm{M}, 2}$ (see open circles in Fig 2), we read in the abscissas a value around $5.7 \times 10^{-6} \mathrm{~mol} \mathrm{~m}^{-3}$ (see vertical solid line) and a $J_{\mathrm{m}}^{\mathrm{SS}}$ around $4.3 \times 10^{-11} \mathrm{~mol} \mathrm{~m}^{-2} \mathrm{~s}^{-1}$.

In order to process data with a simpler expression (and deal with fewer unknown parameters) it is convenient to introduce some approximation into the cubic eqn. (7). One option is to replace the values of $K_{\mathrm{M}, 1}$ and $K_{\mathrm{M}, 2}$ in the denominators of eqn. (7) by a common value. For instance, one could take

$$
K_{\mathrm{M}}=\min \left(K_{\mathrm{M}, 1}, K_{\mathrm{M}, 2}\right)
$$


Fig 2 illustrates that this approximation leads to an underestimation of the solution for $c_{\mathrm{M}}^{\mathrm{SS}}$ (see intersection of the solid line with the markers - , indicated with the vertical dashed line, to the left of exact solution) and thus overestimates the steady-state flux $J_{\mathrm{m}}^{\mathrm{SS}}$. On the other hand, taking $K_{\mathrm{M}}=\max \left(K_{\mathrm{M}, 1}, K_{\mathrm{M}, 2}\right)$ leads to an overestimation of $c_{\mathrm{M}}^{\mathrm{SS}}$.

The mathematical advantage of merging (e.g. by averaging) the $K_{\mathrm{M}^{-}}$values lies in the simplification of the cubic eqn. (7) into a quadratic eqn. whose solution can be written as a kind of modified Best eqn. (van Leeuwen 1999):

$\frac{c_{\mathrm{M}}^{\mathrm{SS}}}{c_{\mathrm{M}}^{*}} \approx \frac{(1-a-b)+\sqrt{(1-a-b)^{2}+4 a}}{2}$

with $a$ being the bioaffinity parameter

$a \equiv \frac{K_{\mathrm{M}}}{c_{\mathrm{M}}^{*}}$

and $b$ the limiting flux ratio

$b \equiv \frac{k_{1} \Gamma_{\max , 1}+k_{2} \Gamma_{\max , 2}}{D_{\mathrm{M}} c_{\mathrm{M}}^{*} / r_{0}}$

The non-linear relationship (especially apparent for large $k_{1}$ and $k_{2}$ ) between $c_{\mathrm{M}}^{\mathrm{SS}}$ and $c_{\mathrm{M}}^{*}$ given by eqn. (9) combined with the Michaelis-Menten relationship between $J_{\mathrm{m}}^{\mathrm{ss}}$ and $c_{\mathrm{M}}^{\mathrm{SS}}$ (associated with the Langmuir adsorption isotherms (2) and (3)) results in a complex relationship between $J_{\mathrm{m}}^{\mathrm{SS}}$ and $c_{\mathrm{M}}^{*}$. An intermediate value of the slope of $J_{\mathrm{m}}^{\mathrm{SS}}$ versus $c_{\mathrm{M}}^{*}$ in between 0 (associated with a zero order kinetics when transporters are saturated ) and 1 (for the first order kinetics and linear adsorption when there is low free metal concentration) seems reasonable in the Langmuirian adsorption context. Thus, if some experimental data exhibit a slope different from 1, this result does not necessarily 
imply, per se, that the present model (diffusion + equilibrium adsorption + first order internalisation kinetics) should be discarded. We can also conclude that, if the experimental $J_{\mathrm{m}}^{\mathrm{SS}}$ vs $c_{\mathrm{M}}^{*}$ plot is not linear (e.g. see bending data in ref. (Slaveykova and Wilkinson 2002)), the linear isotherm model can no longer be sufficient to explain those data and one needs to resort to a Langmuirian approach as done, for instance, in ref. (Slaveykova and Wilkinson 2002) and in this work.

\section{3.- The evolution of fluxes and accumulated amounts towards the steady- state regime}

Fig 3 plots the evolution of the incoming fluxes $J_{\mathrm{m}}$ and $J_{\mathrm{u}}$ with time for some typical parameters from the literature (Wilkinson and Buffle 2004). As expected, the diffusive flux $J_{\mathrm{m}}$ decreases with time and tends towards a steady-state value at which it converges with $J_{\mathrm{u}}$. Notice that the transient regime is very short, so that at around $40 \mathrm{~s}$, all the supplied $J_{\mathrm{m}}$ is internalised as $J_{\mathrm{u}}$. However, the transient regime can explain the sudden increase of both the adsorbed amount $\left(\Gamma=\Gamma_{1}+\Gamma_{2}\right)$ and the total accumulated amount $\left(\Phi_{\mathrm{m}}\right)$, as seen, for the same system, in Fig 4. The results for $\Phi_{\mathrm{m}}$ and $\Phi_{\mathrm{u}}$ in the corresponding linear isotherm (where the linear parameters are computed using eqns. (29) in ref. (Galceran and van Leeuwen 2004)) indicate that saturation has limited the accumulated amounts in the Langmuirian case.

With typical parameters from the literature (taking the largest $k_{1}$-value reported in (Wilkinson and Buffle 2004)), we see in Fig 5 a clear maximum in $J_{\mathrm{u}}$ during the transient regime ( $t \approx 6 \mathrm{~s}$ ). In fact, the existence of a maximum was demonstrated for the linear uptake (Galceran et al. 2004) and has always been found in the numerical experiments with Languirian adsorption. However, this maximum in $J_{\mathrm{u}}$ (which follows 
from a maximum in $\left.c_{\mathrm{M}}\left(r_{0}, t\right)\right)$ is usually so close to the steady-state value that it has little practical relevance.

The overshooting of $c_{\mathrm{M}}\left(r_{0}\right)$ with respect to $c_{\mathrm{M}}^{\mathrm{SS}}$ (i.e. the maximum in the transient of the concentration which is linked to an essentially identical evolution in $J_{\mathrm{u}}$ ) can be understood if diffusion is the predominant phenomenon at short times, while the internalisation kinetics control the flux at long times (Galceran et al. 2004;Galceran and van Leeuwen 2004). Notice that in the overshoot region ( $t>6 \mathrm{~s}$, in Fig 5), $J_{\mathrm{m}}<J_{\mathrm{u}}$ is evident in the figure, while the amount of species adsorbed on the surface is decreasing. In general, for any finite time, the difference $J_{\mathrm{m}}-J_{\mathrm{u}}$ indicates the accumulation (or decrease after the maximum) of $\mathrm{M}$ on the micro-organism surface as adsorbate.

As discussed elsewhere (Galceran et al. 2004;Galceran and van Leeuwen 2004), the approach to steady state can be considerably slow for some parameters, leading to considerable error in the determination of the characteristic parameters if the transient values are taken as the final steady-state value. For instance, Fig 6 shows that the computed $J_{\mathrm{m}}$ for $t=1000 \mathrm{~s}$ is still more than $50 \%$ larger than the eventual $J_{\mathrm{m}}^{\mathrm{ss}}$, in this particular case of a very large number of active receptors. However, the error of taking $J_{\mathrm{m}}$ as $J_{\mathrm{m}}^{\mathrm{SS}}$ is unlikely in practice, if further measurements are carried out, because the experimental values of $J_{\mathrm{m}}$ would not stay constant over a much longer period.

\section{4.- How long does it take to reach steady state?}

Let $t_{\mathrm{SS}}$ denote an estimate of the time for $c_{\mathrm{M}}\left(r_{0}\right)$ to approach $95 \%$ of the steady-state value (the extension to any other prescribed proximity is straightforward). One way of estimating $t_{\mathrm{SS}}$ is to linearise the uptake isotherms (see section 2.2 in ref. (Galceran et al. 
2004)) and replace $J_{\mathrm{m}}$ by $J_{\mathrm{m}}^{\mathrm{dSS}}$. Thus, the balance of fluxes (A-1) becomes the ordinary differential equation

$$
\left(\frac{\Gamma_{\max , 1}}{K_{\mathrm{M}, 1}}+\frac{\Gamma_{\max , 2}}{K_{\mathrm{M}, 2}}\right) \frac{\mathrm{d} c_{\mathrm{M}}\left(r_{0}, t\right)}{\mathrm{d} t}=D_{\mathrm{M}} \frac{c_{\mathrm{M}}^{*}-c_{\mathrm{M}}\left(r_{0}, t\right)}{r_{0}}-\left(\frac{k_{1} \Gamma_{\max , 1}}{K_{\mathrm{M}, 1}}+\frac{k_{2} \Gamma_{\max , 2}}{K_{\mathrm{M}, 2}}\right) c_{\mathrm{M}}\left(r_{0}, t\right)
$$

where the unknown is $c_{\mathrm{M}}\left(r_{0}, t\right)$. Integration from the initial $t=0$ with $c_{\mathrm{M}}\left(r_{0}, t\right)=0$ up to $t_{\mathrm{SS}}$ when $c_{\mathrm{M}}\left(r_{0}, t\right)=0.95 c_{\mathrm{M}}^{\mathrm{SS}}$, and some elementary algebra, using the approximate value for $c_{\mathrm{M}}^{\mathrm{SS}}$ given by (9) and (8), leads to:

$$
\begin{aligned}
t_{\mathrm{SS}}= & \frac{-\left(\frac{\Gamma_{\text {max }, 1}}{K_{\mathrm{M}, 1}}+\frac{\Gamma_{\text {max }, 2}}{K_{\mathrm{M}, 2}}\right)}{\frac{D_{\mathrm{M}}}{r_{0}}+\frac{k_{1} \Gamma_{\max , 1}}{K_{\mathrm{M}, 1}}+\frac{k_{2} \Gamma_{\max , 2}}{K_{\mathrm{M}, 2}}} \times \\
& \times \ln \left(1-\left[1+\frac{r_{0}}{D_{\mathrm{M}}}\left(\frac{k_{1} \Gamma_{\text {max }, 1}}{K_{\mathrm{M}, 1}}+\frac{k_{2} \Gamma_{\text {max }, 2}}{K_{\mathrm{M}, 2}}\right)\right] 0.95 \frac{(1-a-b)+\sqrt{(1-a-b)^{2}+4 a}}{2}\right)
\end{aligned}
$$

The application of eqn. (13) to the data of Fig 3 yields $t_{\mathrm{SS}}=1.9 \mathrm{~s}$, while with the numerical simulation $c_{\mathrm{M}}$ reaches $95 \%$ of $c_{\mathrm{M}}^{\mathrm{SS}}$ at $t=1.8 \mathrm{~s}$, which indicates a good agreement. Notice that, at these times, the supply flux $J_{\mathrm{m}}$ is still very far away from its steady-state value $J_{\mathrm{m}}^{\mathrm{SS}}$. Simple algebra allows to state:

$$
\frac{J_{\mathrm{m}}}{J_{\mathrm{m}}^{\mathrm{SS}}}=0.95+0.05 \frac{D_{\mathrm{M}} c_{\mathrm{M}}^{*}}{r_{0} J_{\mathrm{m}}^{\mathrm{SS}}}
$$

In the particular case of Fig 3, the predictive formula (14) yields a ratio 226 while the one obtained numerically is 117 . The relative disagreement (the order of magnitude is correct, nonetheless) can be due to $c_{\mathrm{M}}^{*}$ in Fig 3 being somewhat high for the linearisation of the isotherms performed in eqn. (12). 
For Fig 6, the numerically obtained $t_{\mathrm{ss}}$ is $44.26 \mathrm{~s}$ and eqn. (13) yields $45.01 \mathrm{~s}$. Formula (14) yields a ratio $J_{\mathrm{m}} / J_{\mathrm{m}}^{\mathrm{sS}}=117$, while the numerically found ratio is 140 .

Fig 7 shows that the estimate given by eqn. (13) is quite reasonable for the trial set of parameters. Other sets of parameters (usually within the range reported in (Wilkinson and Buffle 2004)) also indicate the goodness (not shown here) of the suggested expression. The key conclusion, however, stems from the observation that for the parameters reported in the literature (Wilkinson and Buffle 2004), with $r_{0}$ not larger than $10 \mu \mathrm{m}, t_{\mathrm{ss}}$ is of the order of seconds. This means that the usual experimental measurements (at times longer than 1 minute) will correspond to steady-state conditions and, so, the flux can be computed with the right hand side of expression (7) replacing $c_{\mathrm{M}}(r, t)$ with $c_{\mathrm{M}}^{\mathrm{ss}}$. This supports the use of steady-state expressions for the fluxes, even if a much longer time is required for $J_{\mathrm{m}}$ to approach $J_{m}^{\mathrm{ss}}$ (i.e. a time longer than $J_{\mathrm{u}}$ as expression (14) indicates; see also plot against right-hand axis Y in Fig 7). However, as shown below (and also in ref. (Galceran et al. 2004)) the transient period does have an impact on the accumulated amounts when the system has reached the steady-state regime.

\section{5.- Retrieving parameters from accumulation data}

\subsection{ISSA methodology with total burden}

The simple formulation of the Instantaneous Steady-State Approximation (Galceran et al. 2004) (ISSA) considers that adsorption is practically instantaneous and that steadystate is approached from the very beginning of the experiment. The total amount (or total burden $\Phi_{\mathrm{m}}$ ) outside the transient regime can be estimated as the sum of the 
adsorbed amount (considered to be fixed from the start of the experiment) and the internalised amount (considered to be accumulating at a steady-state rate from the start):

$$
\Phi_{\mathrm{m}}(t) \approx \Gamma^{\mathrm{SS}}+J_{\mathrm{m}}^{\mathrm{SS}} \cdot t
$$

For linear adsorption it has been shown that this procedure is valid for typical parameters (reported in (Slaveykova and Wilkinson 2002)) and can lead to the successful recovery of the unknown parameters (first order internalisation rate constant $k$ and linear adsorption constant, $K_{\mathrm{H}}$ ). Moreover, it has been shown (Galceran et al. 2004;Galceran and van Leeuwen 2004) that the procedure is valid for any set of isotherms at low enough coverage, retrieving a weighted average of the characteristic parameters of the considered isotherms. Here, we assess now the utility of the same approach for the more general case where one kind of site internalises (after a Langmuirian adsorption) and the other kind of site is non-internalising (Langmuir adsorption-only) (Slaveykova and Wilkinson 2002). This model of just one internalising route is tackled by our numerical model by simply putting $k_{2}=0$.

As shown in Fig 8, the fitting of the $\Phi_{\mathrm{m}}$-data to a straight line in the interval $t=11 \mathrm{~s}$ and $t=1000 \mathrm{~s}$ successfully recovers the steady-state flux (slope $=4.73 \times 10^{-12} \mathrm{~mol} \mathrm{~m}^{-2} \mathrm{~s}^{-1}$ representing $99.9 \%$ of the true $J_{\mathrm{m}}^{\mathrm{SS}}$-value) and the total adsorbed amount $\Gamma^{\mathrm{SS}}$ (intercept $=1.92 \times 10^{-7} \mathrm{~mol} \mathrm{~m}^{-2}$ representing $99.9 \%$ of the true $\Gamma^{\mathrm{SS}}$ ). From the fluxes, the product $k_{1} \cdot \Gamma_{\max }$ and $K_{\mathrm{M}, 1}$ can be extracted from the slopes of the plot at different $c_{\mathrm{M}}^{*}$ values. However, the remaining parameters cannot be recovered with confidence from the (correctly found) values of $\Gamma^{\mathrm{SS}}$, because of their large number and coupled impact 
on the final $\Gamma^{\text {SS }}$. When short time experiments provide transient total burden data, they will certainly help in the retrieving of the desired parameters.

\subsection{ISSA methodology with extracted fraction corresponding to that adsorbed onto one type of sites}

Slaveykova and Wilkinson (Slaveykova and Wilkinson 2002) have used a separation procedure to discriminate fractions in the total amount of $\mathrm{Pb}$ taken up by Chlorella kesslerii. The procedure was later applied to the biouptake of $\mathrm{Zn}$ (Hassler and Wilkinson 2003) by the same microalga. The cells were isolated from the experimental medium by filtration and then washed with EDTA. This washing allows quantification of the "extractable" fraction, which they identified as the fraction adsorbed onto the physiologically non-active sites and which we denote as $\Gamma_{2}$ (see Fig 9). Digestion of the cells allowed quantification of the "non-extractable" fraction, including cellular metal and metal adsorbed onto the transporter sites, which we denote as $\Phi_{\mathrm{u}}+\Gamma_{1}$. By plotting the non-extractable fraction versus time and identifying the intercept of the regression line as the amount of metal adsorbed onto the transporter sites, they obtained further information (in addition to $\Gamma_{2}$ obtained from the extractable fraction) which allowed for the determination of all the parameters of the system. We now proceed to assess their methodology with the use of our transient code.

Figs 8 and 10 show the evolution with time of the expected amounts when Slaveykova and Wilkinson parameters are introduced in our transient model. The extractable fraction, following a sudden rise outside the reach of their experimental determination, remains practically constant, indicating that $\Gamma_{2}$ has attained a fixed value which we identify as $\Gamma_{2}^{\mathrm{SS}}$ (the directly computed value is $1.92 \times 10^{-7} \mathrm{~mol} \mathrm{~m}^{-2}$ ). This value, together 
with eqn. (3) provides a new relationship for each $c_{\mathrm{M}}^{*}$, because the internalisation rate constant of Chlorella for lead is low enough to allow $c_{\mathrm{M}}^{\mathrm{sS}}$ to be approximated by $c_{\mathrm{M}}^{*}$ and, so, $\Gamma_{2}^{\mathrm{ss}} \approx \Gamma_{\max , 2} \frac{c_{\mathrm{M}}^{*}}{K_{\mathrm{M}, 2}+c_{\mathrm{M}}^{*}}$. Thus, the parameters $K_{\mathrm{M}, 2}$ and $\Gamma_{\max , 2}$ can be successfully determined.

As seen in Fig 10, the non-extractable fraction also exhibits a sudden rise at very short time and its linear regression, for data corresponding to longer times (i.e. already in the steady-state regime), provides an intercept practically equal to $\Gamma_{1}^{\mathrm{SS}}$ (whose directly computed value is $1.10 \times 10^{-8} \mathrm{~mol} \mathrm{~m}^{-2}$ ). From this regression, the rest of the parameters can be found. We conclude, then, that Slaveykova and Wilkinson methodology is consistent with the transient model developed here and can be successfully used (at least for some set of parameters) in retrieving information from the system.

The rationale behind their approach, which could be seen as another form of Instantaneous Steady-State Approximation, stems from the followings facts: i) steady state is attained very fast, so that all the experimental data correspond to that regime and, in this way, the included adsorption $\Gamma_{1}$ in the non-extractable fraction is just $\Gamma_{1}^{\mathrm{SS}}$. ii) under steady-state conditions the rate of accumulation is just $J_{\mathrm{m}}^{\mathrm{ss}}$.

\subsection{A refined ISSA methodology}

For large enough $t$, the asymptotic expression for the non-extractable amount $\Gamma_{1}+\Phi_{\mathrm{u}}$ can be formalised with the following eqn.

$$
\Gamma_{1}(t)+\Phi_{\mathrm{u}}(t) \approx\left(\Gamma_{1}^{\mathrm{SS}}+\Phi_{\mathrm{u}}\left(t_{0}\right)\right)+J_{\mathrm{m}}^{\mathrm{SS}}\left(t-t_{0}\right)=\left[\Gamma_{1}^{\mathrm{SS}}+\Phi_{\mathrm{u}}\left(t_{0}\right)-J_{\mathrm{m}}^{\mathrm{SS}} \cdot t_{0}\right]+J_{\mathrm{m}}^{\mathrm{SS}} \cdot t
$$


where $t_{0}$ indicates any time once the steady state is approached and $t>t_{0}$. For practical purposes in most experimental methodologies one can consider that $t_{0}$ is the time of the first measurement.

Expression (16) allows to distinguish what could be called the "simple" interpretation of the ISSA intercept from a "refined" interpretation. In the "simple" interpretation, the intercept is just taken as $\Gamma_{1}^{\mathrm{SS}}$, which corresponds to the implicit assumption that the difference $\Phi_{\mathrm{u}}\left(t_{0}\right)-J_{\mathrm{m}}^{\mathrm{SS}} \cdot t_{0}$ is negligible in comparison with $\Gamma_{1}^{\mathrm{SS}}$. Obviously, for some cases, the application of this "simple" ISSA can fail to retrieve the correct parameters. We will discuss, below, some instances of these cases and indicate how a "refined" interpretation based on expression (16) can be useful in extracting acceptable parameters from the intercept experimentally found. In the "refined" ISSA, the approximation just consists in: a) taking the experimental accumulated amounts as truly under steady-state regime (i.e. the term "Instantaneous" in the refined Instantaneous Steady-State Approximation only means that the steady-state regime has settled when the measurements are done) and b) identify the intercept of the linear regression of these data with the intercept of the asymptotic expression (16).

Notice that the intercept of the ISSA regression (for the non-extractable fraction) should correspond to the term in between the square brackets in equation (16), where there is a subtracting term involving $J_{\mathrm{m}}^{\mathrm{SS}}$. If $k_{1}$ is large (even within the range already reported in the literature (Wilkinson and Buffle 2004)), $J_{\mathrm{m}}^{\text {ss }}$ can be large, and a non-detectable delay on the approach to the steady-state can mean that the difference $\Phi_{\mathrm{u}}\left(t_{0}\right)-J_{\mathrm{m}}^{\mathrm{SS}} \cdot t_{0}$ is not negligible in comparison with $\Gamma_{1}^{\mathrm{SS}}$. This is illustrated in Fig 11: the value of the ISSA intercept (found by fitting the regression line in the interval $10 \mathrm{~s}$ to $900 \mathrm{~s}$ ) is 
$1.83 \times 10^{-9} \mathrm{~mol} \mathrm{~m}^{-2}$, while the expected value of $\Gamma_{1}^{\mathrm{SS}}$ is $4.44 \times 10^{-9} \mathrm{~mol} \mathrm{~m}^{-2}$, implying an error of around 59\%. Most likely, this erroneous value would be suspect because the associated value of $K_{\mathrm{M}, 1}$ retrieved from different intercepts at different $c_{\mathrm{M}}^{*}$-values would not match the $K_{\mathrm{M}, 1}$-value retrieved from the slopes of the regressions (i.e. the fluxes). This suspicion could be elucidated by using the numerical code of this work: if the retrieved parameters (with the "simple” interpretation of ISSA) lead to expected values of the non-extractable amount (at several measuring times) different from the amounts found experimentally, then either the model is not suitable or the retrieved parameters are not correct. If we assume that the model is correct, then we can improve the parameter values by numerically finding the unknown $\Gamma_{\max , 1}$ with the "refined" ISSA:

$$
\text { intercept }=\Gamma_{1}^{\mathrm{SS}}+\Phi_{\mathrm{u}}\left(t_{0}\right)-J_{\mathrm{m}}^{\mathrm{SS}} \cdot t_{0}
$$

where $\Gamma_{1}^{\text {SS }}$ is a function of the unknown given by the isotherm in eqn. (2) and $\Phi_{u}$ is also a function of the unknown $\left(\Gamma_{\max , 1}\right)$ via the numerical code. One easy way to solve eqn. (17) consists in following an iterative procedure (where we assume that we know all parameters except $k_{1}$ and $\left.\Gamma_{\max , 1}\right)$ :

i) Take $\Gamma_{1}^{\mathrm{SS}}$ of the first iteration as the intercept (i.e. start applying the "simple" ISSA interpretation).

ii) Apply eqn. (2) in the Steady-State form

$$
\frac{\Gamma_{1}^{\mathrm{SS}}}{\Gamma_{\mathrm{max}, 1}}=\frac{c_{\mathrm{M}}^{\mathrm{SS}}}{K_{\mathrm{M}, 1}+c_{\mathrm{M}}^{\mathrm{SS}}}
$$

and find $\Gamma_{\max , 1}$ of the iteration ( $c_{\mathrm{M}}^{\mathrm{SS}}$ can be computed from (9) taking $k_{2}=0$ ).

iii) Compute $k_{1}$ of the iteration, so that multiplied by the value $\Gamma_{\max , 1}$ of the iteration (found in the previous step) yields the value of the product of the true- $k_{1}$ times the true- 
$\Gamma_{\text {max,1 }}$ (this product is assumed to be known from the regression of the slopes at different $\left.c_{\mathrm{M}}^{*}\right)$.

iv) If the values of $k_{1}$ and $\Gamma_{\text {max, } 1}$ in this iteration are sufficiently close to the previous ones, then we stop the iterative procedure.

v) Run the program of the model and compute the difference $\Phi_{\mathrm{u}}\left(t_{0}\right)-J_{\mathrm{m}}^{\mathrm{SS}} \cdot t_{0}$ for sufficiently long $t_{0}$ (i.e. so that the system is practically under the steady-state regime). Use eqn. (17) to find $\Gamma_{1}^{\mathrm{SS}}$ of the next iteration. Go to step ii).

The application of the iterative procedure to (synthetic) data in Fig 11 is shown in Table 1 , where we see an excellent retrieval of the original parameters $\left(k_{1}=0.43 \mathrm{~s}^{-1}\right.$ and $\Gamma_{\max , 1}$ $=1.50 \times 10^{-7} \mathrm{~mol} \mathrm{~m}^{-2}$ ).

A large number of physiologically active sites can also challenge the applicability of the “simple” ISSA to the extractable fraction (as suggested in (Hassler and Wilkinson 2003) for cells preconditioned in a culture medium with very low concentration of an essential species). Fig 12 depicts the accumulated amounts of a hypothetical extreme case where $\Gamma_{\text {max }, 1}$ has been increased 1000-fold, with $r_{0}=18 \mu \mathrm{m}$ and all other parameters the same as in Fig 10. Notice that the intercept in Fig 12 is a poor estimate of $\Gamma_{1}^{\mathrm{SS}}$. In fact, Fig 12 corresponds to the system shown in section 3 which has not settled to the steady-state regime even at $1000 \mathrm{~s}$ (Fig 6). The iterative procedure (corresponding to the refined ISSA) outlined above recovers the acceptable values $k_{1}=1.48 \times 10^{-4} \mathrm{~s}^{-1}$ and $\Gamma_{\max , 1}=$ $4.37 \times 10^{-4} \mathrm{~mol} \mathrm{~m}^{-2}$ in the fifth iteration. If this system takes so long to approach steady state, then it is self-evident that the "simple" ISSA will be a poor predictor of the system 
parameters. This is highlighted by the data shown in Fig 13, in which a more extreme case is shown and a negative intercept appears.

In general, the predictive capabilities of the "simple” ISSA approach decrease as the size of the micro-organism increases, as expected from the effects of longer times to achieve the steady-state regime. Fig 14 shows the impact of some parameters on the discrepancy between the expected $\Gamma_{1}^{\mathrm{SS}}$ and the "simple” ISSA-retrieved intercept. A remarkably linear dependence on the internalisation rate constant is observed.

\section{6.- Conclusions}

A transient model for biouptake has been developed that takes into account diffusion and Langmuirian adsorption on different internalisation sites followed by first order internalisation. Via its numerical solution or the associated analytical expressions, this model is shown to be useful as an essential step in improving the understanding of the complex characteristics of biouptake and in the design of experiments to determine relevant parameters.

An approximate expression (see eqn. (9)) is obtained for computing the concentration at the cell surface (needed for large internalisation rate constants) from which expressions for the flux follow. This expression also leads to a good estimate (given by eqn. (12) ) for the time needed for $95 \%$ proximity to steady state.

The validity of the steady-state interpretation of the flux at typical measuring times and parameters is demonstrated, while showing the impact of the transient regime on the accumulated amounts. 
The full transient numerical solution developed here is particularly useful to critically assess the potentialities and limitations of ISSA. Retrieving the whole set of parameters of the model just with total-burden steady-state data is difficult, because of the unbalance between a large number of unknown parameters and practically just one straight line of experimental data as the only information.

The "simple" interpretation of the ISSA strategy with additional information due to an extraction procedure is shown to provide good results, so long as the internalisation rate is low. However, we draw attention to possible misleading values of ISSA intercepts, mainly for large internalisation rate constants and/or large radii (for which steady state takes longer to be achieved). In this respect, we highlight equation (16) as helpful for assigning a physical meaning to the intercept of ISSA strategy and the usefulness of having available the full transient model to check for the consistency of the applied methodology and to retrieve the desired parameters from an iterative procedure.

\section{Acknowledgements}

Helpful advice from K.Wilkinson and V. Slaveykova is gratefully acknowledged. The authors also thank the support of this research by the European Commission under contract EVK1-CT-2001-00086 (BIOSPEC; RTD Programme "Preserving the Ecosystem"), and by the Spanish Ministry of Education and Science (DGICYT: Projects BQU2003-9698 and BQU2003-07587) and by the "Comissionat d'Universitats i Recerca de la Generalitat de Catalunya”. 


\section{Appendix A}

We aim at solving the differential equation (1) with the following boundary conditions:

i) flux balance at $r=r_{0}$ :

$$
\frac{d \Gamma_{1}(t)}{d t}+\frac{d \Gamma_{2}(t)}{d t}=D_{\mathrm{M}}\left(\frac{\partial c_{\mathrm{M}}(r, t)}{\partial r}\right)_{r=r_{0}}-k_{1} \Gamma_{1}(t)-k_{2} \Gamma_{2}(t)
$$

ii) semi-infinite diffusion

$$
c_{\mathrm{M}}(r, t)=c_{\mathrm{M}}^{*} \quad r \rightarrow \infty \quad \text { any } t \geq 0
$$

and the initial distribution of $\mathrm{M}$ is assumed to be homogeneous:

$$
c_{\mathrm{M}}(r, t)=c_{\mathrm{M}}^{*} \quad \forall r \quad t=0
$$

The introduction of a new variable

$$
v(r, t) \equiv r c_{\mathrm{M}}(r, t)
$$

allows derivation of a relationship between the flux of $\mathrm{M}$ arriving at the organism surface and its concentration at this surface (Galceran et al. 1998)

$$
\int_{0}^{t}\left(\frac{\partial v(r, \tau)}{\partial r}\right)_{r=r_{0}} d \tau=c_{\mathrm{M}}^{*} t-\frac{1}{\sqrt{\pi D_{\mathrm{M}}}} \int_{0}^{t} \frac{v\left(r_{0}, t\right)-c_{\mathrm{M}}^{*} r_{0}}{\sqrt{t-\tau}} d \tau
$$

which applies for any undisturbed (i.e. following eqn. (1)) spherical semi-infinite diffusion.

Thus, the problem given by the differential eqn. (1) with boundary conditions (A-1)(A-3) can be recast as an integral equation via integration of the flux balance equation $(\mathrm{A}-1)$ : 


$$
\begin{aligned}
\Gamma_{1}(t)+\Gamma_{2}(t)= & \frac{D_{\mathrm{M}}}{r_{0}}\left[c_{\mathrm{M}}^{*} t-\frac{1}{\sqrt{\pi D_{\mathrm{M}}}} \int_{0}^{t} \frac{v\left(r_{0}, \tau\right)-c_{\mathrm{M}}^{*} r_{0}}{\sqrt{t-\tau}} d \tau\right] \\
& -\int_{0}^{t}\left[k_{1} \Gamma_{1}(\tau)+k_{2} \Gamma_{2}(\tau)+\frac{D_{\mathrm{M}} v\left(r_{0}, \tau\right)}{r_{0}^{2}}\right] d \tau
\end{aligned}
$$

The numerical solution of this equation can be obtained by discretizing the unknown $v\left(r_{0}, t\right)$ as a summation of its change in a series of time intervals. A copy of the program in Fortran is available (free of charge) from the authors upon request.

\begin{tabular}{|c|c|c|c|}
\hline Symbol & Definition & Name & Eqns. \\
\hline$a$ & $a \equiv \frac{K_{\mathrm{M}}}{c_{\mathrm{M}}^{*}}$ & bioaffinity parameter & $(10)$ \\
\hline$b$ & & limiting flux ratio & $(11)$ \\
\hline$c_{\mathrm{M}}$ & & $\begin{array}{l}\text { concentration, in } \mathrm{mol} \mathrm{m}^{-3} \text { units, of the } \\
\text { relevant species } \mathrm{M} \text { at a give } r \text { and } t \text {. }\end{array}$ & (1) \\
\hline$c_{\mathrm{M}}^{*}$ & & bulk concentration of $\mathrm{M}$. & (7) \\
\hline$c_{\mathrm{M}}^{\mathrm{SS}}$ & & $\begin{array}{l}\text { steady-state concentration of } \mathrm{M} \text { at the } \\
\text { surface }\left(r=r_{0}\right)\end{array}$ & (9) \\
\hline$D_{\mathrm{M}}$ & & diffusion coefficient of species M & $(1),(5)$ \\
\hline$J_{\mathrm{m}}$ & $D_{\mathrm{M}}\left(\frac{\partial c_{\mathrm{M}}(r, t)}{\partial r}\right)_{r=r_{0}}$ & mass transport (supply) flux & $(5)$ \\
\hline$J^{\mathrm{dSS}}$ & $D_{\mathrm{M}} \frac{c_{\mathrm{M}}^{*}-c_{\mathrm{M}}\left(r_{0}\right)}{r_{0}}$ & diffusive-steady-state flux & $(7)$ \\
\hline$J_{\mathrm{u}}$ & $J_{\mathrm{u}, 1}+J_{\mathrm{u}, 2}$ & $\begin{array}{l}\text { total uptake flux, summation of the } \\
\text { fluxes to each type of site }\end{array}$ & $(4)$ \\
\hline$J_{\mathrm{m}}^{\mathrm{SS}}$ & & steady-state flux (i.e. when $J_{\mathrm{m}}$ & $(15),(16)$ \\
\hline
\end{tabular}

\section{Appendix B: Notation}




\begin{tabular}{|c|c|c|c|}
\hline & & approaches $J_{\mathrm{u}}$ at long times) & \\
\hline$k$ & & $\begin{array}{l}\text { internalisation rate constant for linear } \\
\text { adsorption isotherm }\end{array}$ & \\
\hline$K_{\mathrm{H}}$ & & $\begin{array}{l}\text { Henry adsorption constant for linear } \\
\text { isotherm (in m units ) }\end{array}$ & \\
\hline$K_{\mathrm{M}, 1}\left(K_{\mathrm{M}, 2}\right)$ & & $\begin{array}{l}\text { bioaffinity parameter, in } \mathrm{mol} \mathrm{m}^{-3} \\
\text { units, for sites } 1 \text { (2). }\end{array}$ & (2), (3) \\
\hline$K_{\mathrm{M}}$ & & merged bioaffinity parameter & (8) \\
\hline$k_{1}\left(k_{2}\right)$ & & $\begin{array}{l}\text { internalisation kinetic constant for } \\
\text { sites } 1(2) \text {. }\end{array}$ & (4),(A-1) \\
\hline$r$ & & radial co-ordinate & (1) \\
\hline$t$ & & time variable & (1) \\
\hline$t_{\mathrm{SS}}$ & & $\begin{array}{l}\text { estimate of time so that } \\
c_{\mathrm{M}}\left(r_{0}\right) \geq 0.95 c_{\mathrm{M}}^{\mathrm{ss}}\end{array}$ & (13) \\
\hline$v$ & $r c_{\mathrm{M}}(r, t)$ & auxiliary variable & $(\mathrm{A}-4)$ \\
\hline$\Gamma_{1}\left(\Gamma_{2}\right)$ & & $\begin{array}{l}\text { surface concentration of } \mathrm{M} \text { at sites of } \\
\text { type } 1(2) .\end{array}$ & $(2),(3)$ \\
\hline$\Gamma_{\max , 1}\left(\Gamma_{\max , 2}\right)$ & & $\begin{array}{l}\text { maximum surface concentration of M } \\
\text { at sites of type } 1 \text { (2). }\end{array}$ & (2), (3) \\
\hline$\Gamma_{1}^{\mathrm{SS}}\left(\Gamma_{2}^{\mathrm{SS}}\right)$ & & $\begin{array}{l}\text { steady-state value of adsorbed amount } \\
\text { on sites of kind } 1 \text { (2). }\end{array}$ & \\
\hline$\Phi_{\mathrm{m}}$ & $\Phi_{\mathrm{m}}(t) \equiv \int_{0}^{t} J_{\mathrm{m}}(\tau) d \tau$ & \begin{tabular}{|l} 
accumulated supplied amount \\
\end{tabular} & (6) \\
\hline$\Phi_{\mathrm{u}}$ & $\Phi_{\mathrm{u}}(t) \equiv \int_{0}^{t} J_{\mathrm{u}}(\tau) d \tau$ & cumulative uptake amount & (6) \\
\hline
\end{tabular}




\section{References}

Campbell, P. G. C. 1995, Interactions between Trace Metal and Aquatic Organisms: A Critique of the Free-ion Activity Model, in Tessier, A., Turner, D. R. (Eds), Metal Speciation and Bioavailability in Aquatic Systems, John Wiley \& Sons, Chichester, pp. 45-102.

Campbell, P. G. C., Errecalde, O., Fortin, C., Hiriart-Baer, W. R., Vigneault, B., 2002. Metal bioavailability to phytoplankton - applicability of the biotic ligand model. Comp.Biochem.Physiol.C 133, 189-206.

Fortin, C., Campbell, P. G. C., 2000. Silver uptake by the green alga Chlamydomonas reinhardtii in relation to chemical speciation: Influence of chloride.

Environ.Toxicol.Chem. 19, 2769-2778.

Galceran, J., Monné, J., Puy, J., van Leeuwen, H. P., 2004. The impact of the transient flux on biouptake accumulation. Linear adsorption and first-order internalisation coupled with spherical semi-infinite mass transport. Mar.Chem. 85, 89-102.

Galceran, J., Salvador, J., Puy, J., Mas, F., Gimenez, D., Esteban, M., 1998. Amalgamation effects in reverse pulse polarography at spherical electrodes. Influence on speciation measurements. J.Electroanal.Chem. 442, 151-167.

Galceran, J., van Leeuwen, H. P. 2004, Dynamics of biouptake processes: the role of transport, adsorption and internalisation, in van Leeuwen, H. P., Koester, W. (Eds), Physicochemical kinetics and transport at chemical-biological surfaces., vol. 9 John Wiley, Chichester, UK, pp. 147-203.

Hassler, C. S., Slaveykova, V. I., Wilkinson, K. J., 2004b. Discriminating between intra- and extracellular metals using chemical extractions. Macromolecular Chemistry and Physics 2, 237-247.

Hassler, C. S., Slaveykova, V. I., Wilkinson, K. J., 2004a. Some fundamental (and often overlooked) considerations underlying the free ion activity and biotic ligand models. Environ.Toxicol.Chem. 23, 283-291.

Hassler, C. S., Wilkinson, K. J., 2003. Failure of the biotic ligand and free-ion activity models to explain zinc bioaccumulation by Chlorella kesslerii. Environ.Toxicol.Chem. 22, 620-626.

Hudson, R. J. M., 1998. Which aqueous species control the rates of trace metal uptake by aquatic biota? Observations and predictions of non- equilibrium effects. Sci.Total Envir. 219, 95-115.

Hudson, R. J. M., Morel, F. M. M., 1990. Iron transport in marine-phytoplankton kinetics of cellular and medium coordination reactions. Limnol.Oceanogr. 35, 10021020.

Jansen, S., Blust, R., van Leeuwen, H. P., 2002. Metal speciation dynamics and bioavailability: Zn(II) and Cd(II) uptake by mussel (Mytilus edulis) and carp (Cyprinus carpio). Environ.Sci.Technol. 36, 2164-2170. 
Lippemeier, S., Hintze, R., Vanselow, K. H., Hartig, P., Colijn, F., 2001. In-line recording of PAM fluorescence of phytoplankton cultures as a new tool for studying effects of fluctuating nutrient supply on photosynthesis. Eur.J.Phycol. 36, 100.

Pinheiro, J. P., Galceran, J., van Leeuwen, H. P., 2004. Metal speciation dynamics and bioavailability: Bulk depletion effects. Environmental Science \& Technology 38, 23972405.

Slaveykova, V. I., Wilkinson, K. J., 2002. Physicochemical aspects of lead bioaccumulation by Chlorella vulgaris. Environ.Sci.Technol. 36, 969-975.

Sunda, W. G., Huntsman, S. A., 1992. Feedback interactions between zinc and phytoplankton in seawater. Limnol.Oceanogr. 37, 25-40.

Tessier, A., Buffle, J., Campbell, P. G. C. 1994, Uptake of Trace Metals by Aquatic Organisms, in Buffle, J., DeVitre, R. R. (Eds), Chemical and Biological Regulation of Aquatic Systems, Lewis Publishers, Boca Raton, FL, pp. 197-230.

van Leeuwen, H. P., 1999. Metal speciation dynamics and bioavailability: Inert and labile complexes. Environ.Sci.Technol. 33, 3743-3748.

van Leeuwen, H. P., Pinheiro, J. P., 2001. Speciation dynamics and bioavailability of metals. Exploration of the case of two uptake routes. Pure Appl.Chem. 73, 39-44.

Whitfield, M., Turner, D. R. 1979, Critical assessment of the relationship between biological thermodynamic and electrochemical availability, in Jenne, E. A. (Ed.), Chemical Modeling in Aqueous Systems, American Chemical Society, Washington DC, pp. 657-680.

Wilkinson, K. J., Buffle, J. 2004, Critical evaluation of physicochemical parameters and processes for modeling the biological uptake of trace metals in environmental (aquatic) systems, in van Leeuwen, H. P., Koester, W. (Eds), Physicochemical kinetics and transport at chemical-biological surfaces., vol. 9 John Wiley, Chichester, UK, pp. 445533.

Wilkinson, K. J., Slaveykova, V. I., Hassler, C. S., Rossier, C., 2002. Physicochemical mechanisms of trace metal bioaccumulation by microorganisms. Chimia 56, 681-684. 


\section{Figures}

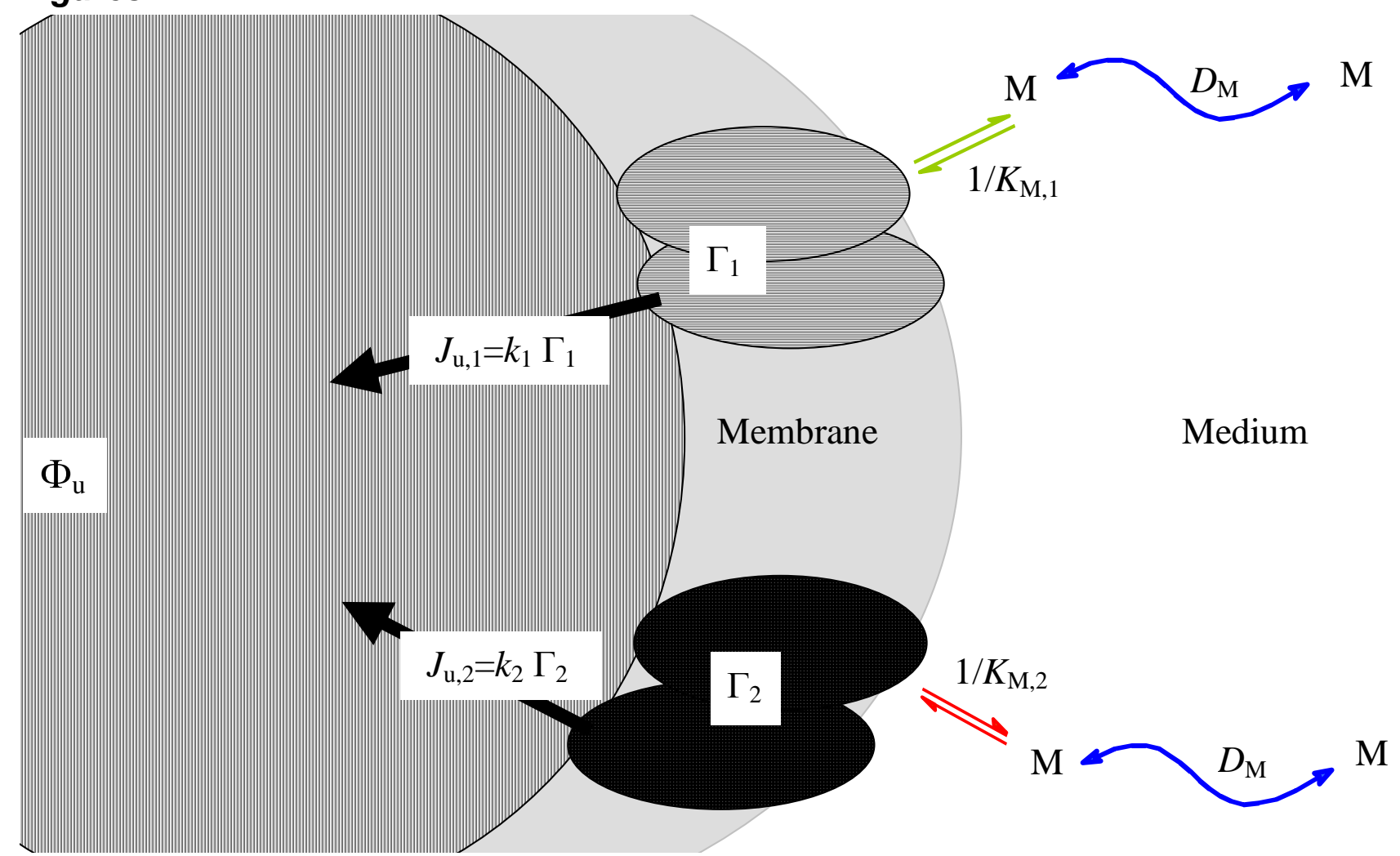

Fig 1: Outline of the uptake model showing the spherical diffusion of species $M$ through the medium towards 2 different internalisation sites where Langmuirian adsorption can be followed by internalisation. The radius of the micro-organism is $r_{0}$. $\Phi_{\mathrm{u}}$ is the internalised amount (inside the cell). 


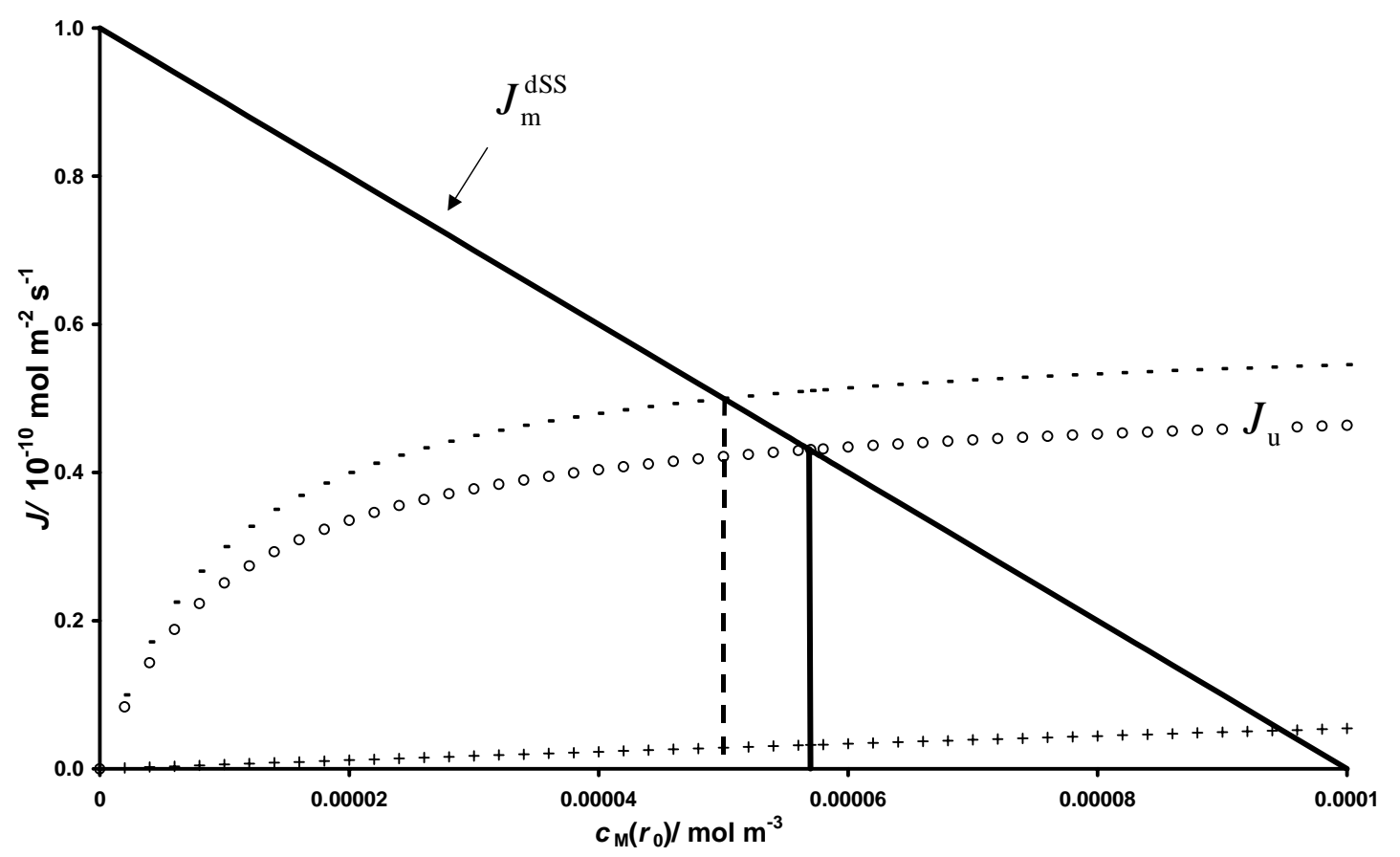

Fig 2 : Fluxes vs. $c_{\mathrm{M}}\left(r_{0}\right)$ plot showing the application of the graphical procedure in 3 different cases with Langmuirian adsorption. The straight line with negative slope indicates the diffusive flux $J_{\mathrm{m}}^{\mathrm{dSs}}$ (defined in Appendix B). Markers stand for the uptake flux $J_{\mathrm{u}}$ for the three considered systems: o (Open circle): $K_{\mathrm{M}, 1}=10^{-5} \mathrm{~mol} \cdot \mathrm{m}^{-3}$ and $K_{\mathrm{M}, 2}=10^{-3} \mathrm{~mol} \cdot \mathrm{m}^{-3}$;- (minus) ): $K_{\mathrm{M}, 1}=K_{\mathrm{M}, 2}=10^{-5}$ mole $\mathrm{m}^{-3} ;+$ (plus) $K_{\mathrm{M}, 1}=K_{\mathrm{M}, 2}=10^{-3}$ mole $\mathrm{m}^{-3}$. The ordinate of the intersection of the continuous line yields the $J_{\mathrm{m}}^{\mathrm{SS}}$-value, while its abscissa is $c_{\mathrm{M}}^{\mathrm{SS}}$. Other parameters: $D_{\mathrm{M}}=10^{-9} \mathrm{~m}^{2} \mathrm{~s}^{-1}, c_{\mathrm{M}}^{*}=10^{-4} \mathrm{~mol} \cdot \mathrm{m}^{-3}, r_{0}=1$ $\mathrm{mm}, k_{1}=10^{-2} \mathrm{~s}^{-1}, k_{2}=10 \mathrm{~s}^{-1}, \Gamma_{\max , 1}=10^{-8} \mathrm{~mol} \cdot \mathrm{m}^{-2}$ and $\Gamma_{\max , 2}=10^{-12} \mathrm{~mol} \cdot \mathrm{m}^{-2}$. 


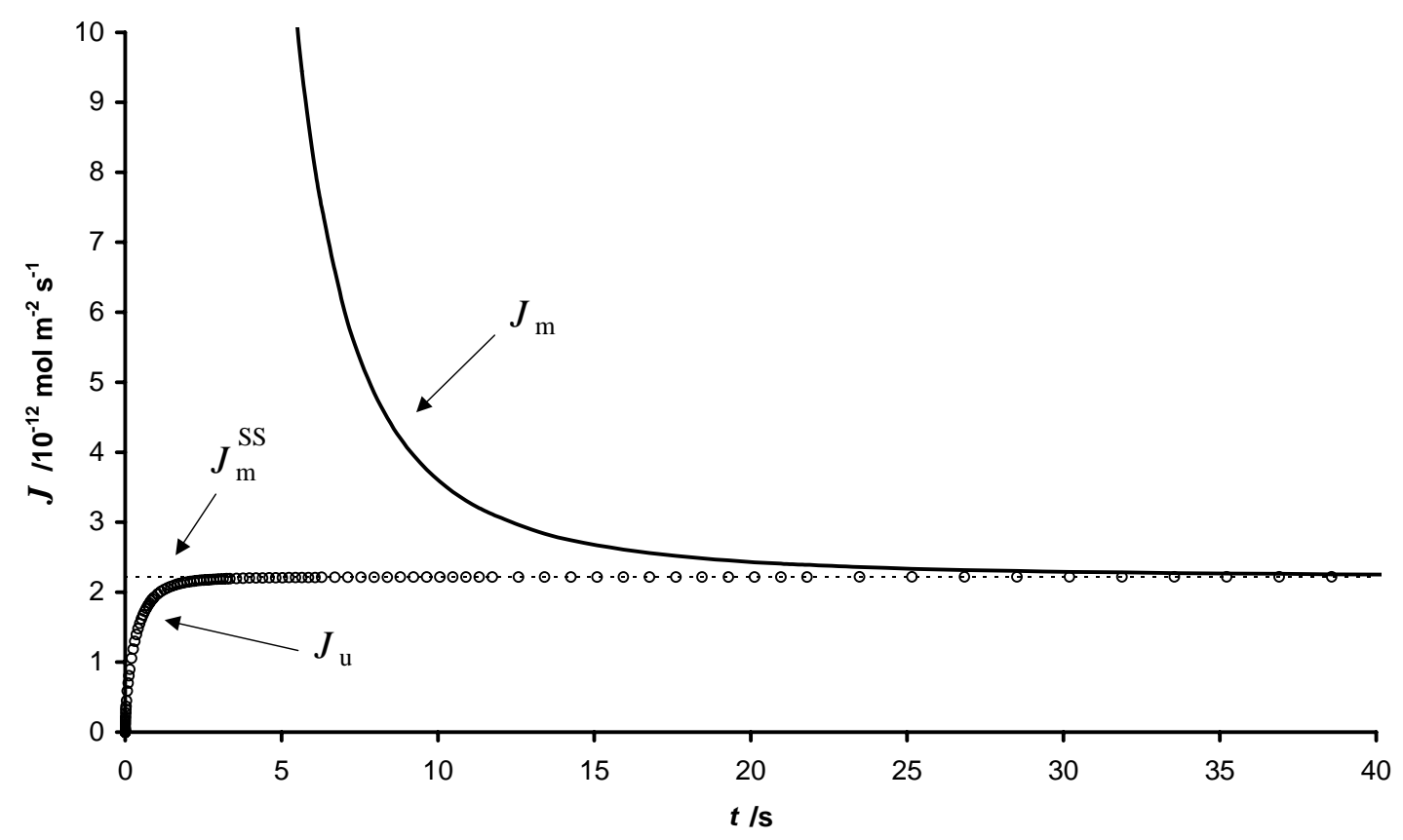

Fig 3: Evolution of the fluxes in the early stages of the uptake process. Both $J_{\mathrm{m}}$ (solid line) and $J_{\mathrm{u}}$ (marker circle o) eventually merge in $J_{\mathrm{m}}^{\mathrm{SS}}$ (dashed line). Parameters: $D_{\mathrm{M}}=10^{-9} \mathrm{~m}^{2} \mathrm{~s}^{-1}, c_{\mathrm{M}}^{*}=10^{-4} \mathrm{~mol} \mathrm{~m}^{-3}, r_{0}=10 \mu \mathrm{m}, K_{\mathrm{M}, 1}=3.98 \times 10^{-6} \mathrm{~mol} \mathrm{~m}^{-3}, K_{\mathrm{M}, 2}=6.3 \times 10^{-4}$ $\mathrm{mol} \mathrm{m}^{-3}, \Gamma_{\text {max }, 1}=1.5 \times 10^{-12} \mathrm{~mol} \mathrm{~m}^{-2}, \Gamma_{\max , 2}=4 \times 10^{-8} \mathrm{~mol} \mathrm{~m}^{-2}, k_{1}=2 \times 10^{-2} \mathrm{~s}^{-1}$ and $k_{2}=4 \times 10^{-4}$ $\mathrm{s}^{-1}$. 


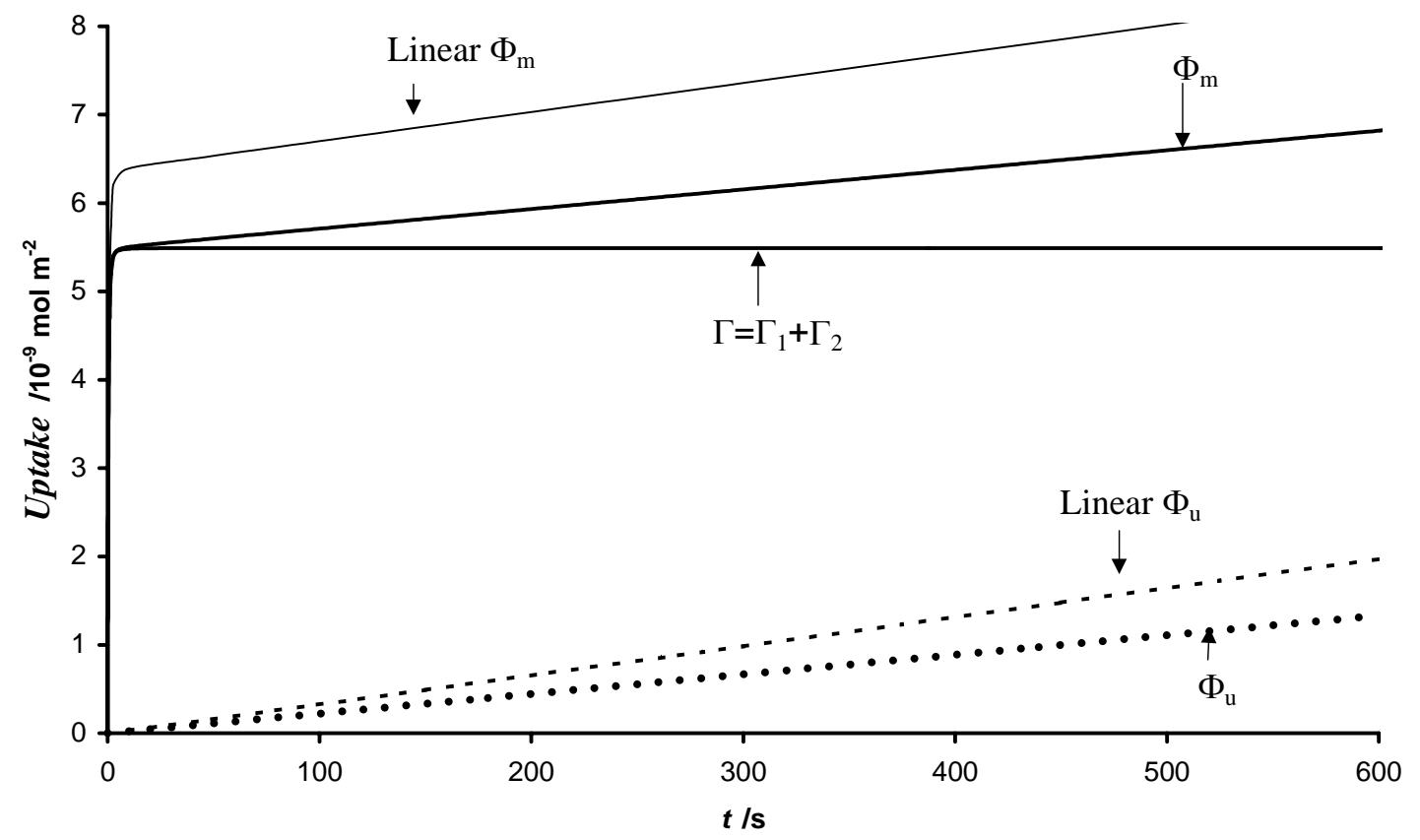

Fig 4: Evolution of the accumulated amounts in the early stages of the uptake process, showing the sudden rise of the adsorbed and total amounts. $\Phi_{\mathrm{m}}$ indicates the total burden supplied (internalised plus adsorbed); the internalised (cellular) amount $\Phi_{\mathrm{u}}$ is shown in the dotted line; the adsorbed amount is $\Gamma=\Gamma_{1}+\Gamma_{2}$. Same parameters as in previous figure. 


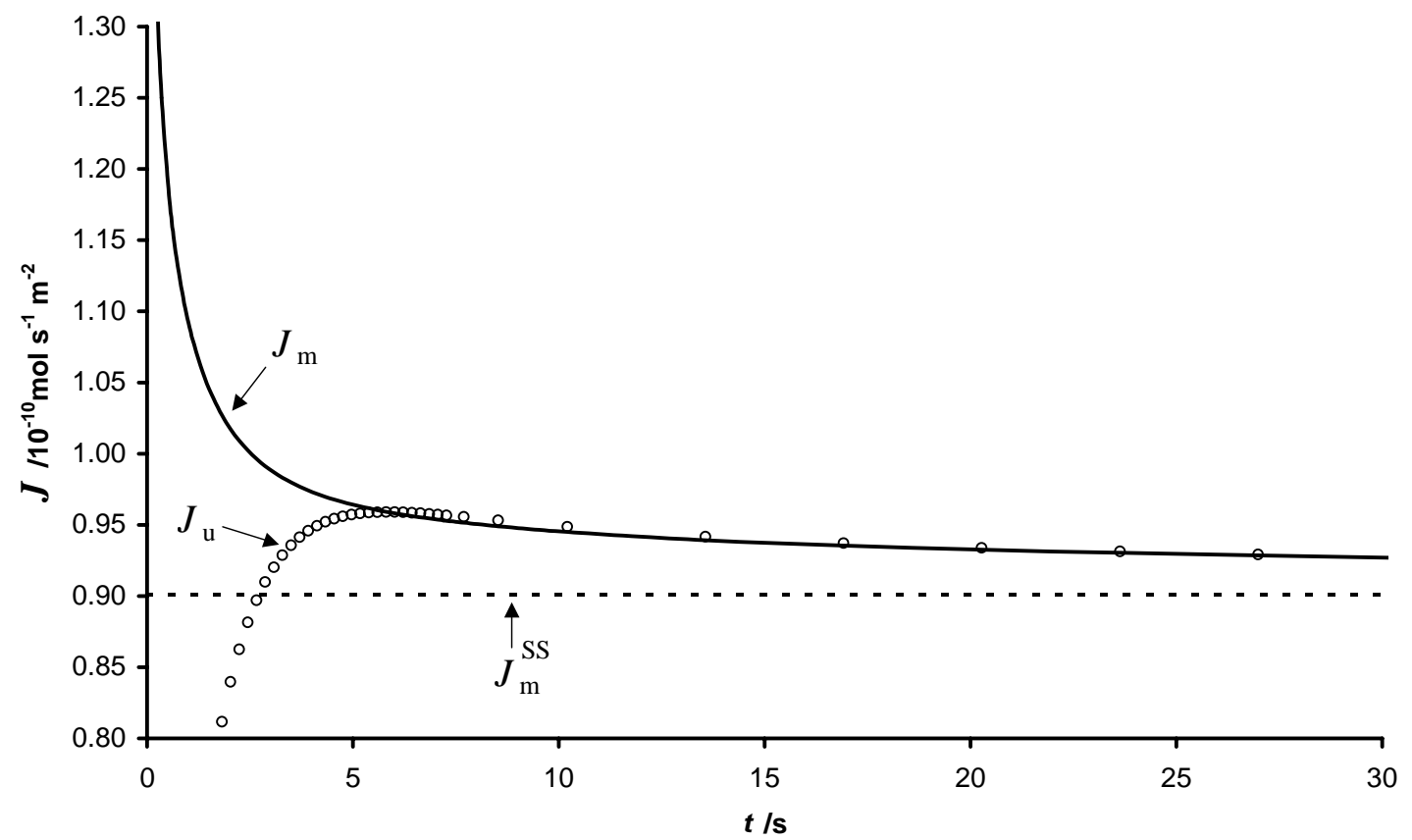

Fig 5: Flux evolution showing a clear maximum in $J_{\mathrm{u}}$ (marker circle o). The supply flux $J_{\mathrm{m}}$ (solid line) just decreases with increasing time. The horizontal dashed line indicates the eventual steady-state flux $J_{\mathrm{m}}^{\mathrm{ss}}$. Parameters: $D_{\mathrm{M}}=10^{-9} \mathrm{~m}^{2} \mathrm{~s}^{-1}, c_{\mathrm{M}}^{*}=10^{-6} \mathrm{~mol} \cdot \mathrm{m}^{-3}, r_{0}=10$ $\mu \mathrm{m}, K_{\mathrm{M}, 1}=10^{-6} \mathrm{~mol} \cdot \mathrm{m}^{-3}, K_{\mathrm{M}, 2}=10^{-4} \mathrm{~mol} \cdot \mathrm{m}^{-3}, \Gamma_{\max , 1}=10^{-9} \mathrm{~mol} \cdot \mathrm{m}^{-2}, \Gamma_{\max , 2}=4 \times 10^{-8} \mathrm{~mol} \cdot \mathrm{m}^{-2}$, $k_{1}=1 \mathrm{~s}^{-1}$ and $k_{2}=10^{-4} \mathrm{~s}^{-1}$. 


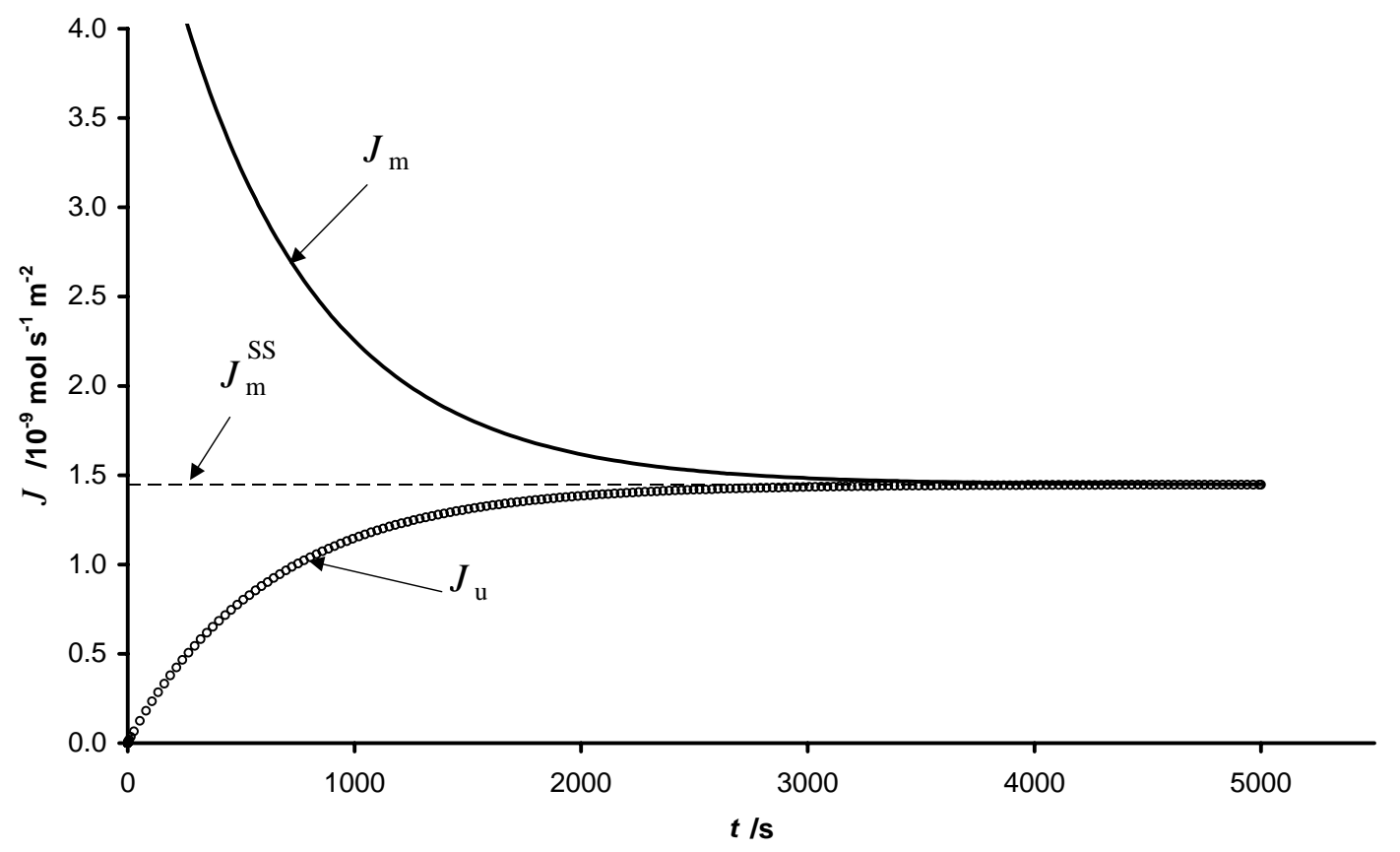

Fig 6: Slow evolution of $J_{\mathrm{m}}$ and $J_{\mathrm{u}}$ towards the steady-state flux $J_{\mathrm{m}}^{\mathrm{SS}}$. Parameters: $D_{\mathrm{M}}=9.45 \times 10^{-10} \mathrm{~m}^{2} \mathrm{~s}^{-1}, \quad c_{\mathrm{M}}^{*}=10^{-4} \mathrm{~mol} \mathrm{~m}^{-3}, \quad r_{0}=18 \mu \mathrm{m}, \quad K_{\mathrm{M}, 1}=3.16 \times 10^{-3} \mathrm{~mol} \mathrm{~m}^{-3}$, $K_{\mathrm{M}, 2}=8.9 \times 10^{-3} \mathrm{~mol} \mathrm{~m}{ }^{-3}, \Gamma_{\max , 1}=1.5 \times 10^{-4} \mathrm{~mol} \cdot \mathrm{m}^{-2}, \Gamma_{\max , 2}=6.6 \times 10^{-6} \mathrm{~mol} \mathrm{~m}^{-2}, k_{1}=4.3 \times 10^{-4}$ $\mathrm{s}^{-1}$ and $k_{2}=0$. 


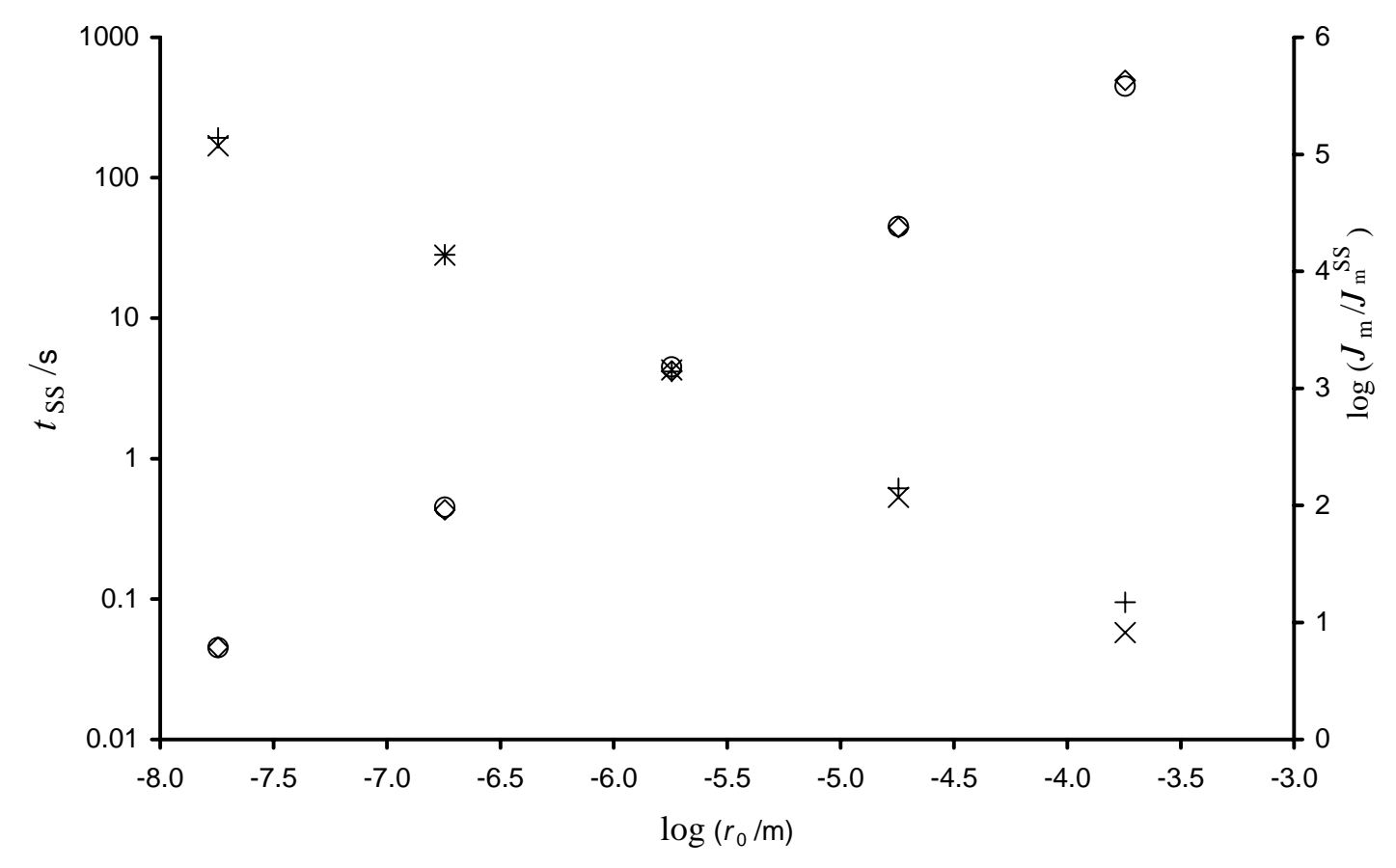

Fig 7: Time needed for $c_{\mathrm{M}}\left(r_{0}, t\right)$ to reach $95 \%$ of the steady-state value $c_{\mathrm{M}}^{\mathrm{SS}}$ ( $t_{\mathrm{SS}}$, plotted against the left-hand side ordinate axis) in front of the radius $\left(r_{0}\right)$. Diamonds stand for the numerical solution with our program while circles are computed with the approximate expression (13). Against the right-hand side Y-axis, we have plotted the ratio $J_{m} / J_{m}^{\mathrm{SS}}$ at the time $t_{\mathrm{SS}}$ (given by the numerical solution) as found from the numerical calculations (marker + ) or applying expression (14) (cross $\times$ marker). Parameters: $\quad D_{\mathrm{M}}=9.45 \times 10^{-10} \mathrm{~m}^{2} \mathrm{~s}^{-1}, \quad c_{\mathrm{M}}^{*}=2.5 \times 10^{-4} \mathrm{~mol} \mathrm{~m}^{-3}, \quad K_{\mathrm{M}, 1}=3.16 \times 10^{-3} \mathrm{~mol} \mathrm{~m}^{-3}$, $K_{\mathrm{M}, 2}=8.9 \times 10^{-3} \mathrm{~mol} \cdot \mathrm{m}^{-3}, \Gamma_{\max , 1}=1.5 \times 10^{-7} \mathrm{~mol} \mathrm{~m}^{-2}, \Gamma_{\max , 2}=6.6 \times 10^{-6} \mathrm{~mol} \mathrm{~m}^{-2}, k_{1}=4.3 \times 10^{-4}$ $\mathrm{s}^{-1}$ and $k_{2}=0$. 


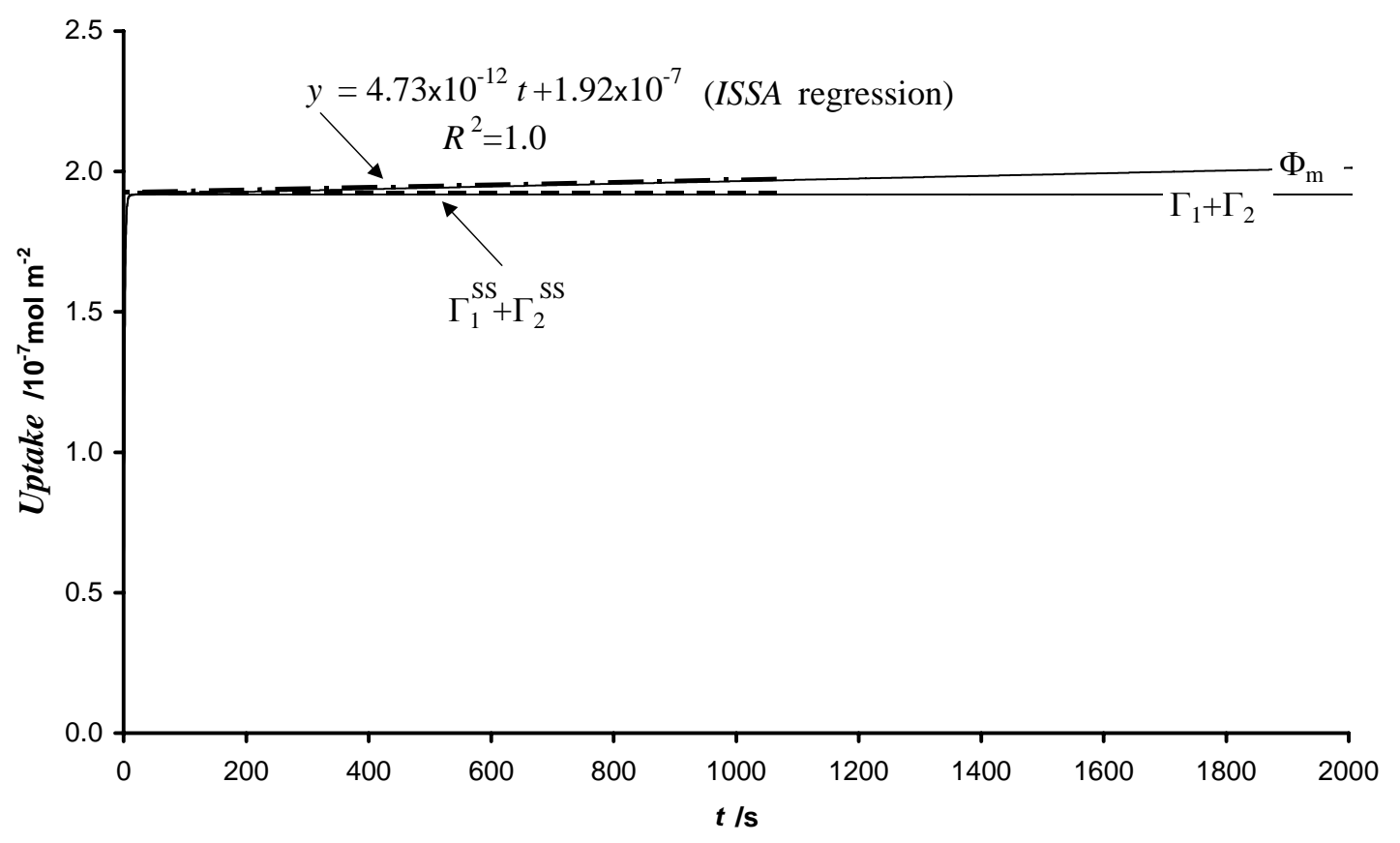

Fig 8 The application of the Instantaneous Steady State Approximation (ISSA) to totalburden data successfully retrieves $\Gamma^{\mathrm{SS}}$ (see dashed line) via the intercept of the linear regression (see dotted-dashed line). However further information is needed for the recovery of all the parameters of the system. Parameters: $D_{\mathrm{M}}=9.45 \times 10^{-10} \mathrm{~m}^{2} \mathrm{~s}^{-1}$, $c_{\mathrm{M}}^{*}=2.5 \times 10^{-4} \mathrm{~mol} \mathrm{~m}^{-3}, r_{0}=1,8 \mu \mathrm{m}, K_{\mathrm{M}, 1}=3.16 \times 10^{-3} \mathrm{~mol} \mathrm{~m}^{-3}, K_{\mathrm{M}, 2}=8.9 \times 10^{-3} \mathrm{~mol} \mathrm{~m}^{-3}$, $\Gamma_{\text {max }, 1}=1.5 \times 10^{-7} \mathrm{~mol} \mathrm{~m}^{-2}, \Gamma_{\max , 2}=6.6 \times 10^{-6} \mathrm{~mol} \mathrm{~m}^{-2}, k_{1}=4.3 \times 10^{-4} \mathrm{~s}^{-1}$ and $k_{2}=0$. 


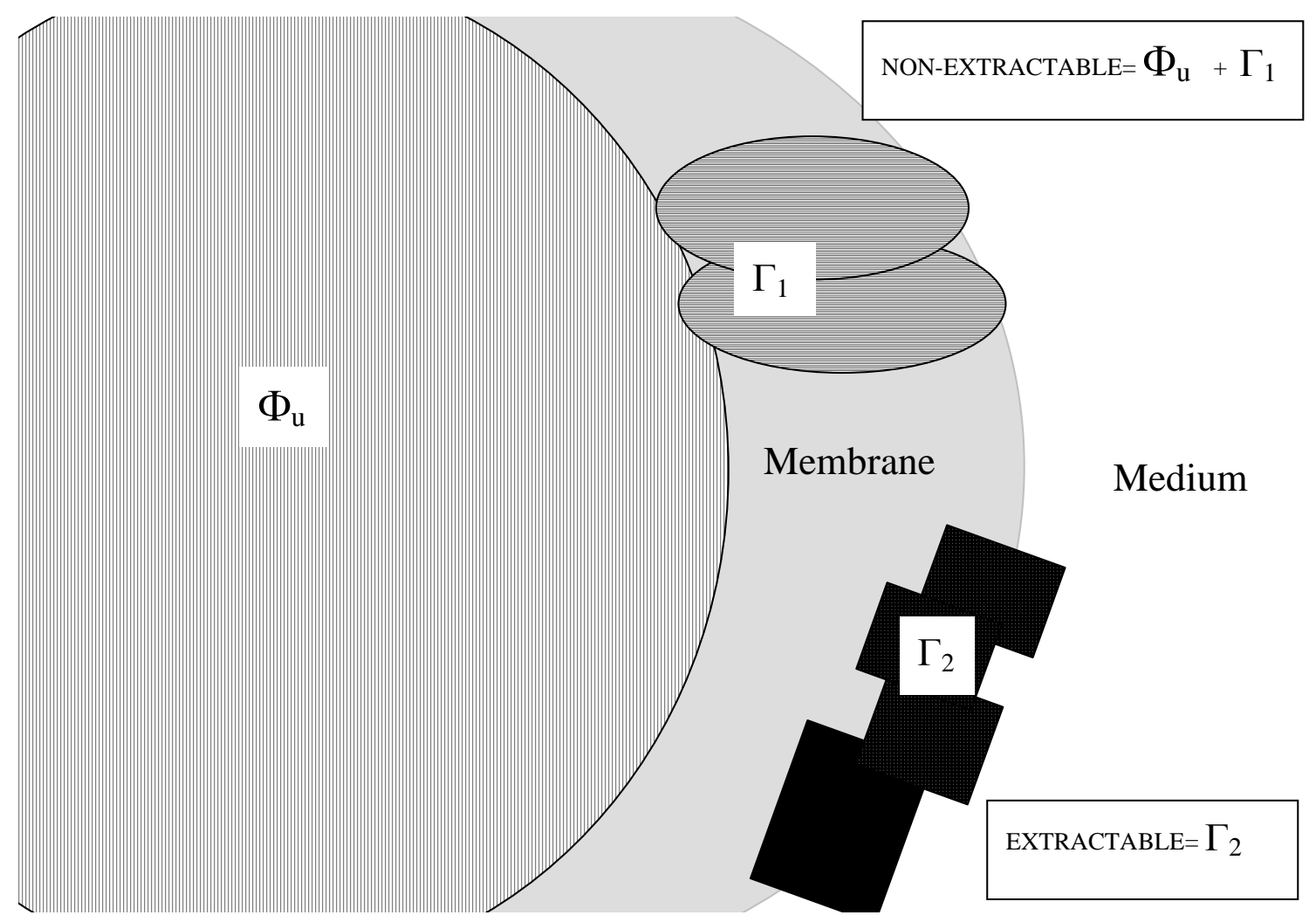

Fig 9 Outline of the interpretation of the extraction procedure by Wilkinson and coworkers (Hassler et al. 2004b;Hassler et al. 2004a;Hassler and Wilkinson 2003;Slaveykova and Wilkinson 2002; Wilkinson et al. 2002) in the nomenclature of this work. The total burden $\left(\Phi_{\mathrm{m}}\right)$ is the sum of extractable and non-extractable. Extractable is considered to be the adsorbed amount on the non-active sites $\left(\Gamma_{2}\right)$. Nonextractable is considered to be the sum of the internalised amount $\left(\Phi_{\mathrm{u}}\right.$, which could be also called "cellular") and the adsorbed amount on the active sites $\left(\Gamma_{1}\right)$. 


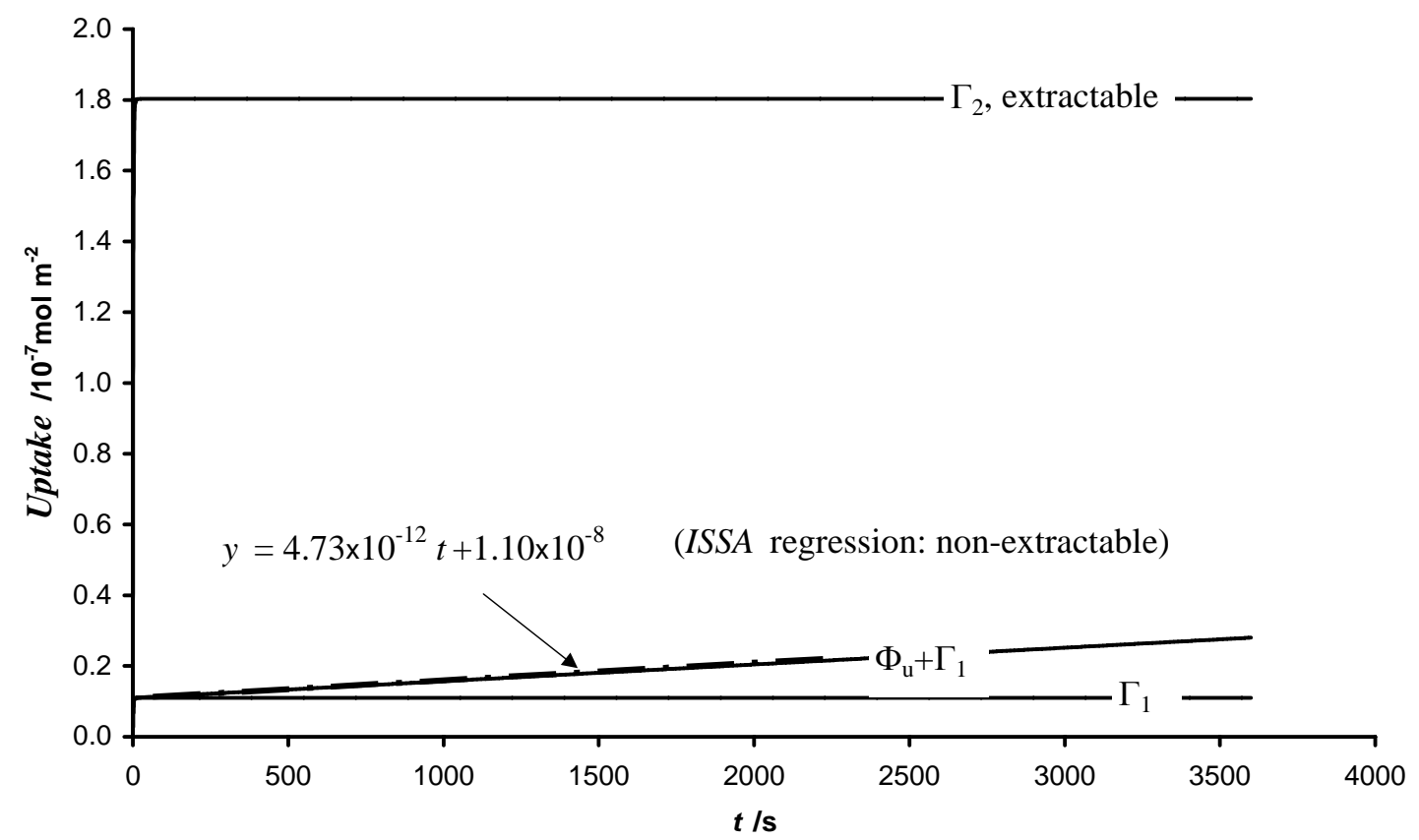

Fig 10: Successful application of ISSA with data corresponding to the non-extractable fraction: the intercept provides $\Gamma_{1}^{\mathrm{SS}}$ (Slaveykova and Wilkinson 2002). The practically constant value of the extractable fraction $\left(\Gamma_{2}\right.$, in the upper part of the plot) provides $\Gamma_{2}^{\mathrm{SS}}$. Same parameters as in Fig 8. 


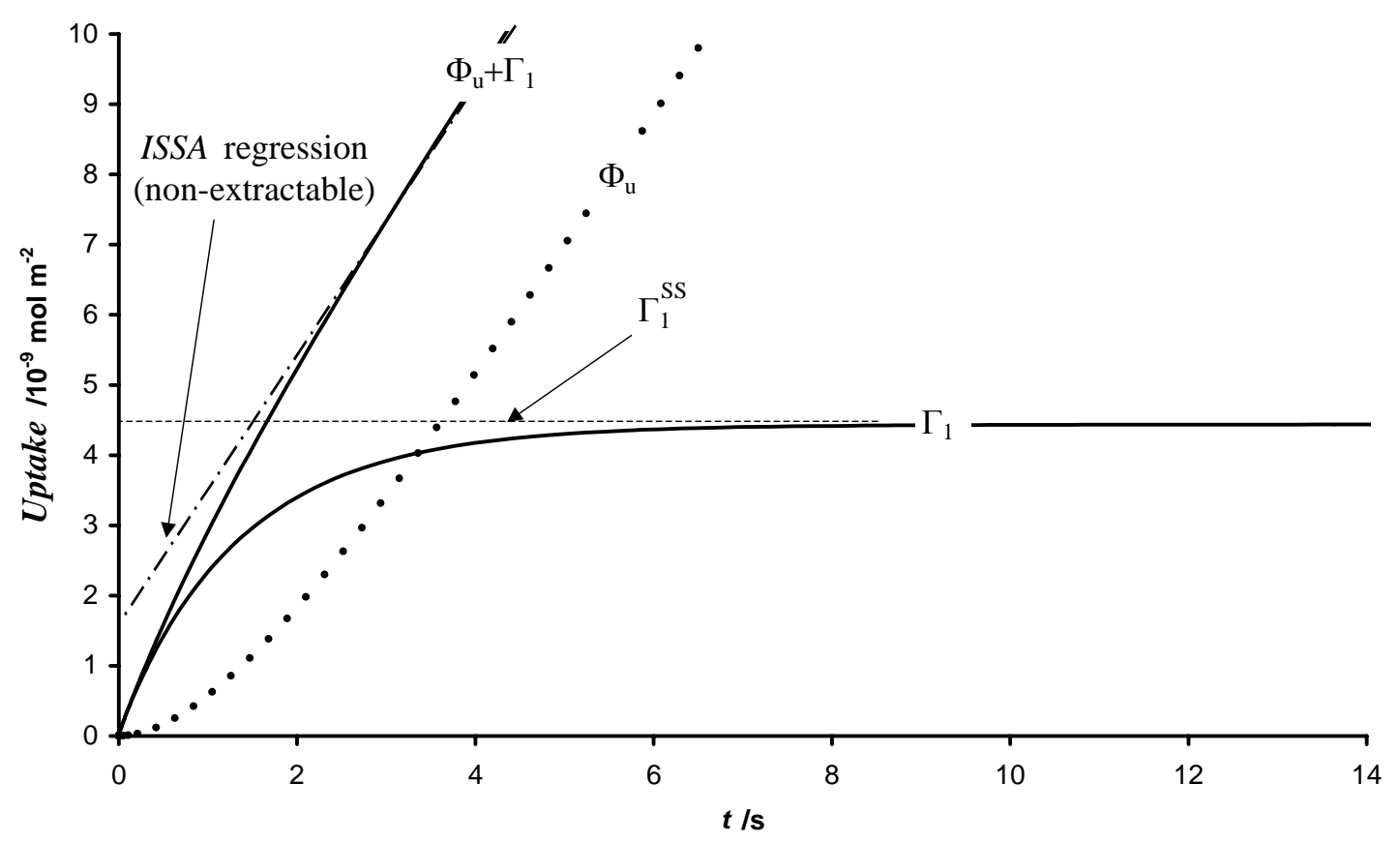

Fig 11 Example of the misleading application of ISSA with a "simple” interpretation, due to the impact of the transient on the accumulated amounts, even when steady-state is already established. The upper continuous line corresponds to the simulated experimental data of the non-extractable amount. The intercept of a linear regression fitting steady-state values is much lower than the true value $\Gamma_{1}^{\mathrm{sS}}$ (see horizontal dashed line). Same parameters as in Fig 8 except $c_{\mathrm{M}}^{*}=10^{-4} \mathrm{~mol} \mathrm{~m}^{-3}$ and $k_{1}=0.43 \mathrm{~s}^{-1}$. 


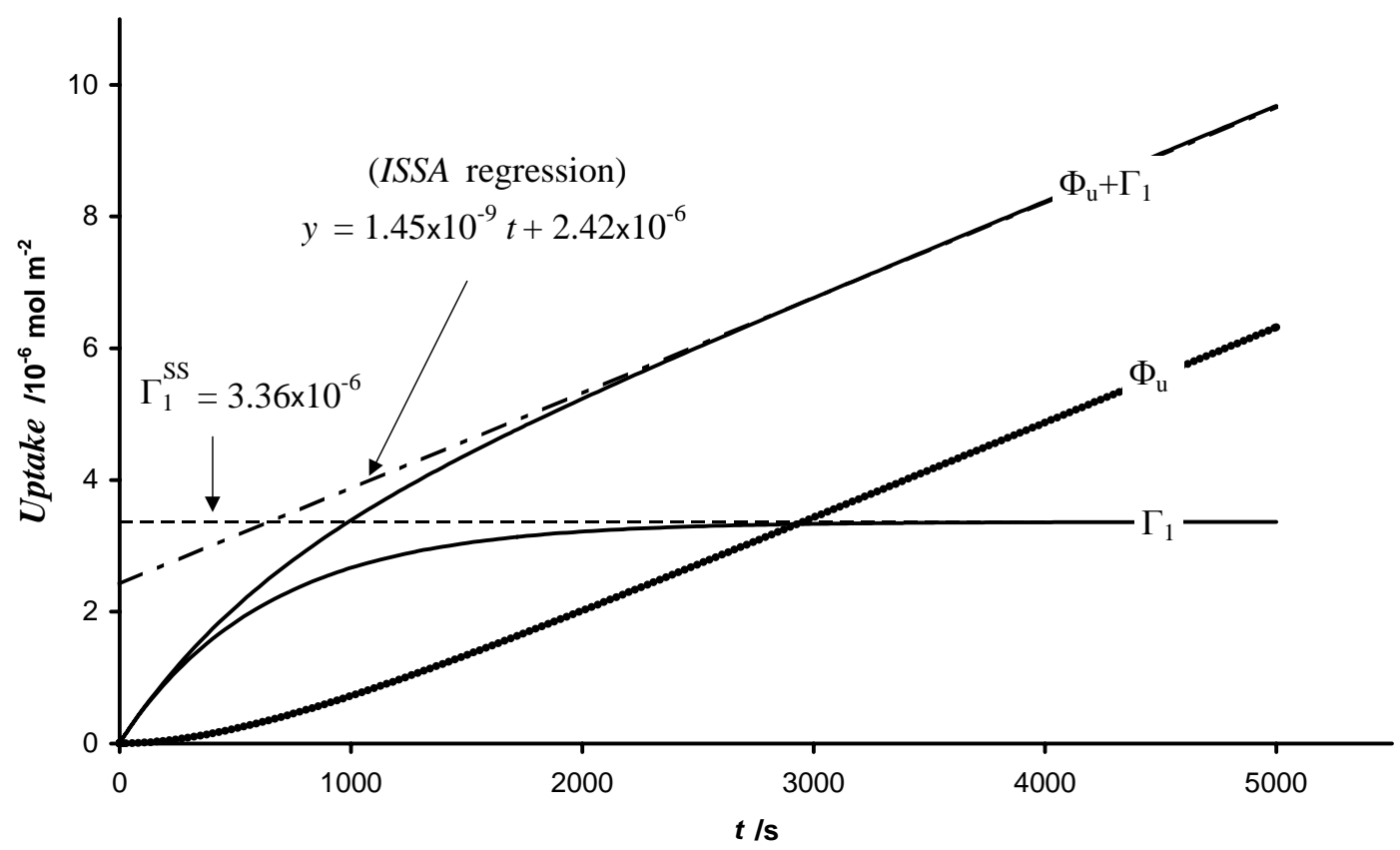

Fig 12 As explained in section 5, a large number of transporter sites $\left(\Gamma_{\text {max }, 1}\right)$ can yield an ISSA intercept of the regression of non-extractable amounts not representing the steady-state adsorbed amount $\Gamma_{1}^{S S}$. Parameters as in Fig 6. 


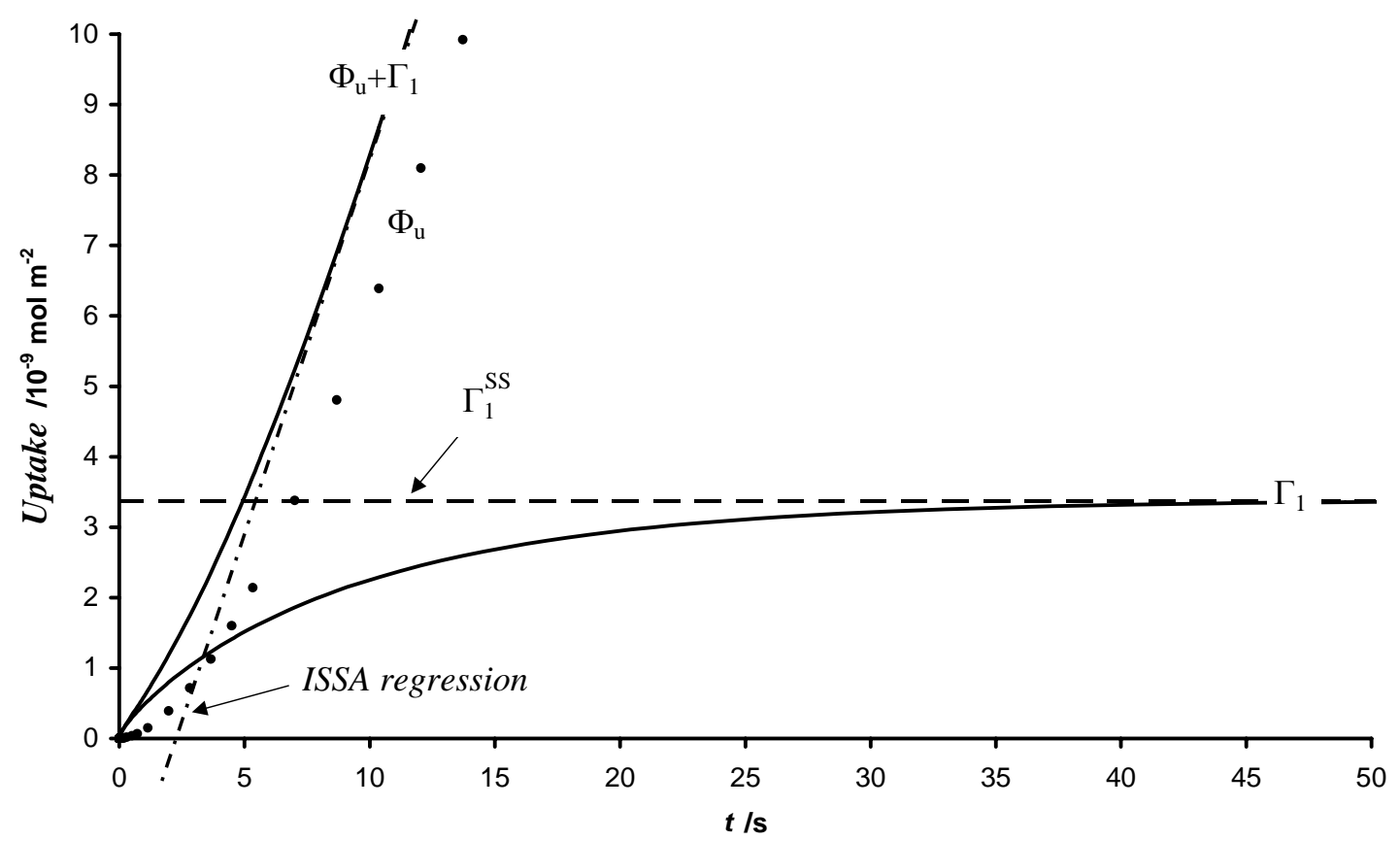

Fig 13 Clear breakdown of ISSA methodology with "simple" interpretation for the same parameters as in Fig 11 except a larger radius $\left(r_{0}=18 \mu \mathrm{m}\right)$ yielding a negative intercept. Same markers as in previous figures. 


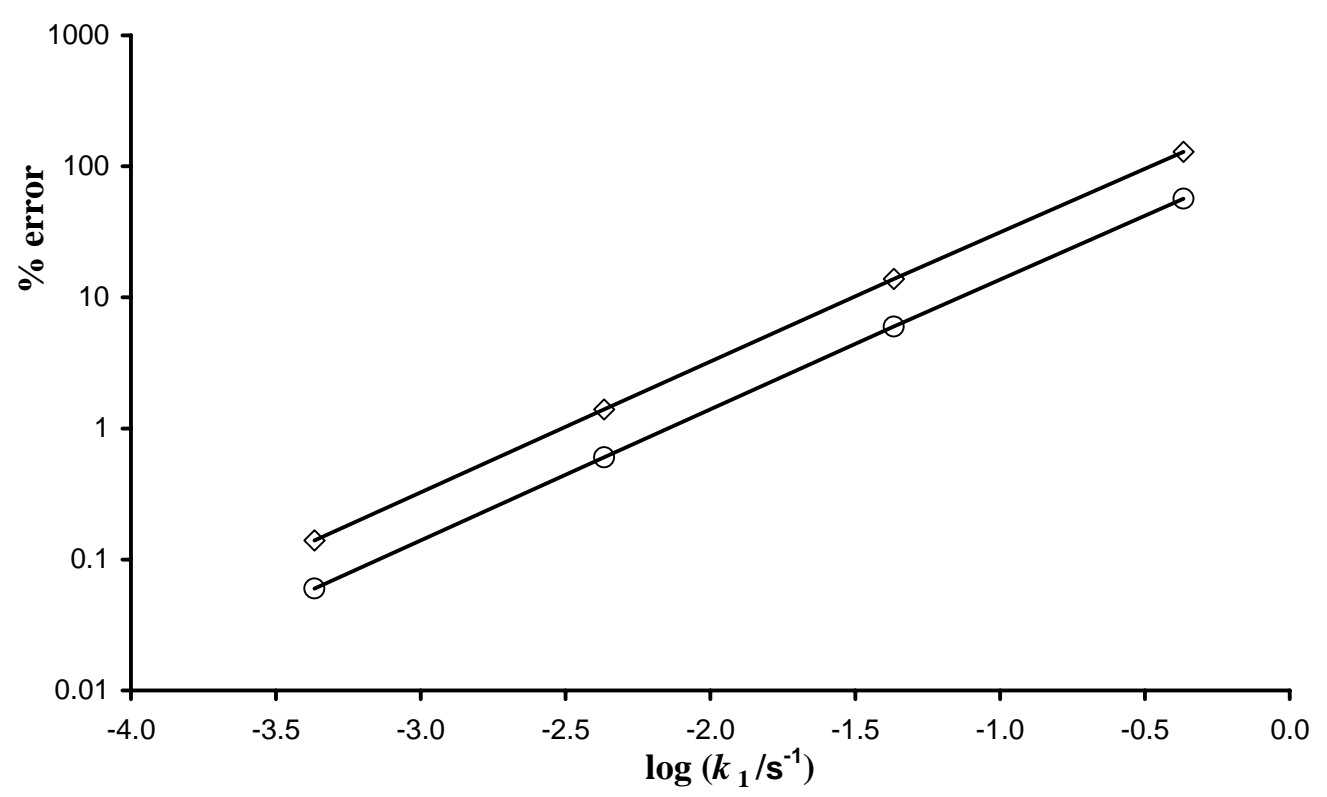

Fig 14 Plot of the percentage of error in the determination of $\Gamma_{1}^{\mathrm{sS}}$ from recovered intercepts (ISSA methodology with "simple" interpretation) as a function of the internalisation rate constant $k_{1}$ for 2 different radii $(1.8 \mu \mathrm{m}$ in circle o and $18 \mu \mathrm{m}$ in diamonds). Parameters as in Fig 8 with $c_{\mathrm{M}}^{*}=2.5 \times 10^{-4} \mathrm{~mol} \mathrm{~m}^{-3}$. 
Table 1: Values of the parameters obtained along the iterative procedure corresponding to the refined ISSA applied to data from Fig 11. The values of $\Phi_{\mathrm{u}}\left(t_{0}\right)-J_{\mathrm{m}}^{\mathrm{SS}} \cdot t_{0}$ given by the numerical code at $t_{0}$ come from the region $2700 \mathrm{~s}-$ 3000 s where they were constant up to 2 decimal places.

\begin{tabular}{|l|l|l|l|l|l|}
\hline & first iteration & second & third iteration & fourth & fifth iteration \\
& & iteration & & iteration & \\
\hline$\Phi_{\mathrm{u}}\left(t_{0}\right)-J_{\mathrm{m}}^{\mathrm{SS}} \cdot t_{0}$ & 0 (simple ISSA) & $-2.48 \times 10^{-9}$ & $-2.58 \times 10^{-9}$ & $-2.59 \times 10^{-9}$ & $-2.58 \times 10^{-9}$ \\
\hline$\Gamma_{1}^{\mathrm{SS}} / \mathrm{mol} \mathrm{m}^{-2}$ & $1.83 \times 10^{-9}$ & $4.31 \times 10^{-9}$ & $4.41 \times 10^{-9}$ & $4.42 \times 10^{-9}$ & $4.41 \times 10^{-9}$ \\
\hline$\Gamma_{\text {max }, 1} / \mathrm{mol} \mathrm{m}^{-2}$ & $6.18 \times 10^{-8}$ & $1.46 \times 10^{-7}$ & $1.49 \times 10^{-7}$ & $1.49 \times 10^{-7}$ & $1.49 \times 10^{-7}$ \\
\hline$k_{1} / \mathrm{s}^{-1}$ & 1.04 & 0.44 & 0.43 & 0.43 & 0.43 \\
\hline
\end{tabular}

